\title{
Genetic Algorithms at UC Davis/LLNL
}

Compiled by

V. Rao Vemuri

Fall 1993 


\section{Preface}

This volume contains six papers, an introduction and an appendix. These six were selected from eight papers written and submitted by students describing their term projects for the first course in Genetic Algorithms at the University of California at Davis/Livermore during the Fall Quarter 1993 (EAL 216G: Special Topics in Computer Science: Genetic Algorithms and Optimization).

The introduction and the appendix are written by the course instructor, Prof. V. Rao Vemuri. The introduction contains a very brief introduction to the subject of genetic algorithms and the appendix contains the course outline and other supporting material used during the course.

This is the first time this course was taught at the University of California, Davis. The class was taught simultaneously from both Livermore and Davis via our television link. Lectures were delivered from Davis on Tuesdays and from Livermore on Thursdays. Six of the students were at Davis and two at Livermore.

The students who participated in this course barely had six weeks from the time their proposals were approved to the time the papers were due. During this period, they were simultaneously learning the subject. The reader is urged not to read too much into the papers. Almost all the papers are necessarily incomplete. The intent of this collection is keep a record of this experiment, that is all. Of course many students are continuing to improve their papers and bring them to publishable format.

Acknowledgment: The instructor wishes to acknowledge the assistance he received from Dr. John Koza of Stanford University during the preparation for this course. Thanks are also due Dr. John Feo of the Lawrence Livermore National Laboratory for the help received in producing this report.

V. Rao Vemuri

Professor

Department of Applied Science

P. O. Box 808, L-794

University of California at Davis

Livermore, CA 94550 
Table of Contents

1. Genetic Algorithms: An Introduction 5

2. Training Neural Networks with Genetic Algorithms for Character Classification 7

3. Genetic Algorithm Based Information Filtering 15

4. DNA Restriction Fragment Map Assembly with Genetic Algorithm:

5. Protein Folding and Genetic Algorithms: Torsional Energy Minimization

6. The Node Embedding Problem:

A Genetic Algorithm based Approach 45

7. A Study of Genetic Algorithms to find Approximate Solutions to Hard 3CNF Problems

8. Appendix: Relevant Course Material 


\title{
Genetic Algorithms: An Introduction
}

\author{
V. Rao Vemuri \\ Professor, Department of Applied Science \\ University of California at Davis \\ Livermore, CA 94550 \\ vemuri@icdc.IInl.gov
}

\section{Abstract}

A tutorial introduction to genetic algorithms is given. This brief tutorial should serve the purpose of introducing the subject to the novice. The tutorial is followed by a brief commentary on the term project reports that follow.

\section{Introduction:}

A genetic algorithm (GA) can be thought of as a process that is analogous to evolution by natural selection, but a process that takes place in a computer. The nature of the processes taking place in a genetic algorithm are best understood by way of an example involving optimization. Consider the problem of finding the maximum (or, for that matter, maxima) of a function $f(x)$ in the interval $[0,1]$. Instead of using the traditional calculus methods, we wish to use a genetic algorithm. Toward this goal, we first pick a population of $n$ random points in the said interval and evaluate the fitness of each of these points as a solution to the given problem, namely finding the value of $x$ at which $f(x)$ is a maximum. As we do not have a priori knowledge of the solution, the best we can do at this stage is to select a subset of the $n$ points where the solution appears to have some desired properties in satisfying the fitness criterion. Using these selected points as "parents", a GA develops "offspring" solutions which are presumably better in satisfying the fitness criterion. The process continues for several generations until an acceptable solution is found. This, in a nutshell, is what a genetic algorithm is all about. 
The word "genetic" in a genetic algorithm, at first sight, is metaphorical. The computational steps involved in the "algorithm" are loosely based on ideas from population genetics. In mathematical parlance, this carl be thought of as a randomized parallel search method. Genetic algorithms allow us to use a computer to evolve solutions over time and evaluate them rapidly while the evolution and evaluation (by proceses like reproduction, inheritance, competition, selection and mutation) in the natural world may take millions of years.

At this point, closer look at the optimization problem, introduced in the first paragraph, would be instructive. For the sake of simplicity, let us assume that the function $f(x)$ is evalauted at ten randomly selected points, say $x 1, \times 2, \ldots \times 10$ in $[0,1]$. These ten individual values can be represented, in the binary format, by ten distinctly different strings of zeros and ones. In GA parlance, these binary strings are called chromosomes, and each bit in a chromosome is a gene. A chromosome refers to an individual solution. When one talks about, say, the fitness of chromosome xi, one really means the value $f(x i)$. If only chromosomes with high fitness are selected for reproduction, then that process is called fitness proportionate reproduction scheme. Other schemes can be defined. Selected parents are allowed to produce offspring. The chromosomal composition of the offspring is determined by crossing over the chromosomes of its two parents. Occassionally, mutation can be used to randomly introduce new traits into an individual (solution). This process can be repeated for several generations (iterations) until a suitable stopping criterion is satisfied. This metaphor can be continued to a fine degree of detail.

While designing a genetic algorithm to solve a specific problem, two important decisions must be made. The so-called representation problem deals with the mapping between the binary chromosomal strings and the candidate solutions. The second is the definition of the fitness function. In the maximization of $f(x)$, both these issues are trivially solved. In general, this is not always this obvious. In complicated problems, say the nonlinear transportation problem, it is very difficult to define a linear binary string to represent a chromosome. Then, it is far better to modify the definitions of chromosome, crossover, mutation, etc. to suit the structure of the problem. 


\title{
Training Neural Networks with Genetic Algorithms for Character Classification
}

\author{
Karen A. Norris \\ Department of Electrical \& Computer Engineering \\ University of California, Davis \\ Davis, California 95616 \\ norris@ece.ucdavis.edu
}

\begin{abstract}
Algorithms for training artificial neural networks such as backpropagation, often employ some form of gradient descent in the search for an optimal weight set. The problem associated with these algorithms is their tendency to converge to local minima, or not to converge at all. The techniques of genetic search (i.e. genetic algorithms) are applied to training a neural network for classification of character data. Testing of the network reveals that genetic algorithms may be somewhat effective for neural network training, but results are somewhat mixed.
\end{abstract}

\section{Introduction}

The success of gradient descent training algorithms depends on the continuity and quality of the search space. The problems associated with these algorithms usually result from a failure of the algorithm to converge (Hush and Horne, 1993). This especially holds true when the search space is convoluted with local minima or other traps, such as extensive plateaus with zero gradient. Furthermore, the final point of descent of the search space may completely miss the global minimum thus producing only a local optimum solution. One advantage to utilizing GAs for training networks is that they perform global search procedures and make no assumption about the problem space being searched. Therefore, the problematic behavior of a gradient descent search technique stalling is eliminated, since GAs do not require gradient information for determining network weights.

This study investigates the application of genetic algorithms to the problem of optimizing the weight set of a two-layer, feed-forward perceptron neural network. The network was trained to classify a pseudo-random, jointly Gaussian data set of 1000 eight-dimensional feature vectors which represented the characters $A, B, C$, and $D$ (Marill and Green, 1963). The performance of the genetically generated network weights was compared to the neural network trained using a gradient descent algorithm known as back-propagation. 


\section{Two-layer Perceptron Neural Network}

The neural network selected for this study was a two-layer, feed-forward perceptron consisting of eight input nodes $\left(x_{0}, x_{1}, \ldots, x_{7}\right)$, four output nodes $\left(y_{0}, y_{1}, \ldots, y_{3}\right)$, and one hidden layer comprised of fifteen internal nodes $\left(x_{0}^{\prime}, x_{1}^{\prime}, \ldots, x_{14}^{\prime}\right)$. Each of the network nodes were fully connected; every node in the input layer is connected to every node in the hidden layer and every node in the hidden layer is connected to every node in the output layer (see Figure 1). Thus, the input data vector is fed into the input layer, the output of this layer is then fed into the hidden layer whose output is fed into the output layer. The four output nodes $y_{0}, y_{1}, y_{2}$, and $y_{3}$ correspond to the four characters A, B, C, and D, respectively, defined within our classification problem.

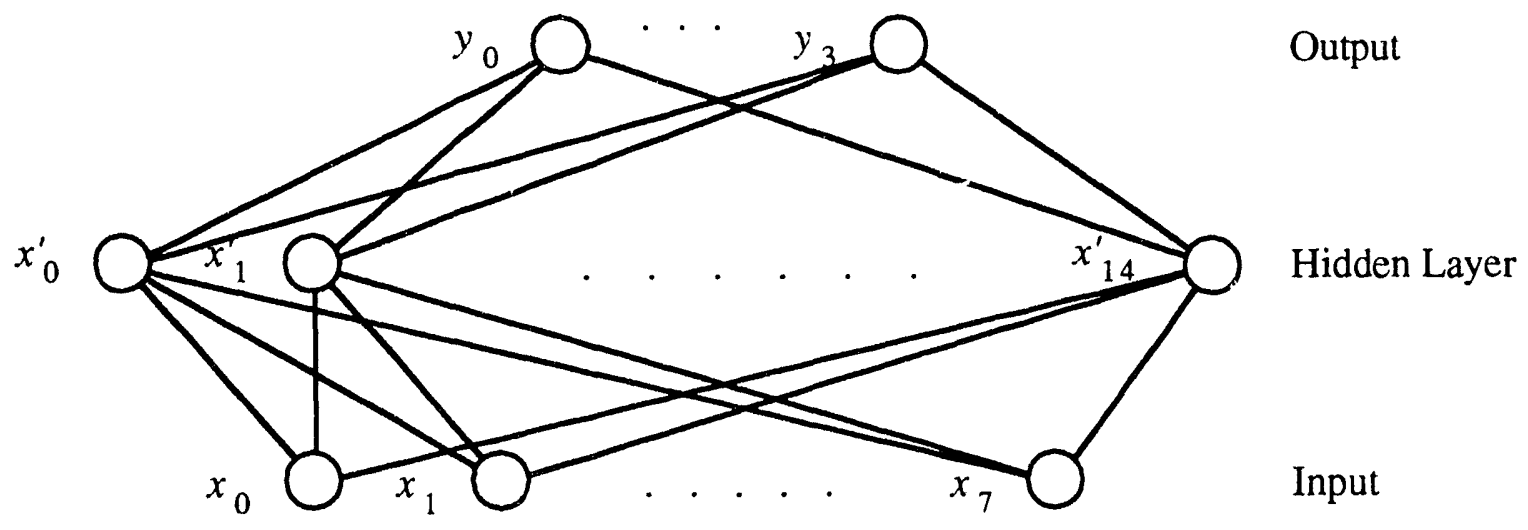

Figure 1. Two-Layer Perceptron

The two-layer perceptron network forms a weighted sum of the eight components of the input data vector and subtracts an internal threshold, $\theta$, (i.e., off-set value) as follows

$$
\sum_{i=0}^{7} w_{i j} x_{i}-\theta_{j} \forall j \in[0, \ldots, 14]
$$

where $\mathrm{w}_{i j}$ represents the connection strengths from the input nodes to the hidden layer (i.e., input weights) and $x_{i}$ represents the input layer nodes. These results are then passed through a sigmoid nonlinearity

$$
f(\lambda)=\frac{1}{1+e^{-(\lambda-\theta)}}
$$

which varies monotonically from 0 to 1 as $\lambda$ varies from $-\infty$ to $\infty$. The output of the hidden layer nodes $x_{j}^{\prime}$ are calculated as follows

$$
x_{j}^{\prime}=f\left(\sum_{i=0}^{7} w_{i j} x_{i}-\theta_{j}\right) \forall j \in[0, \ldots, 14]
$$


Similarly, the network output values $y_{k}$ were calculated by

$$
y_{k}=f\left(\sum_{j=0}^{14} w_{j k}^{\prime} x_{j}^{\prime}-\theta_{k}^{\prime}\right) \forall k \in[0, \ldots, 3],
$$

where $w_{j k}^{\prime}$ represents the connection strengths from the hidden layer nodes to the output layer (i.e., output weights) and $x_{j}^{\prime}$ represents the hidden layer nodes as previously defined.

Performance of the network is evaluated by presenting each of the 1000 test vectors to the network for classification. The output node with the largest value was then used to determine the assigned class of the data vector. If output node $y_{0}$ contained the largest value, then the data vector was classified as the character $\mathrm{A}$; if the largest value was found in output node $y 1$, then the data vector was classified as a B; etc. After the data vectors were classified, the fitness of the network was then computed as the overall probability of error of assigning each data vector to its correct class. This was accomplished by comparing the actual class of the data vector with the network assigned class.

\section{Back-Propagation Training Algorithm}

Network training was accomplished using a back-propagation algorithm to minimize the cost function, $c_{f}$,

$$
c_{f}=\sum_{k=0}^{3} \frac{1}{2}\left(d_{k}-y_{k}\right)^{2}
$$

where $d_{k}$ represents the desired network outputs and $y_{k}$ represents the actual network outputs. The desired output of all nodes is typically low unless that node corresponds to the actual class of the current input vector in which case it is high. The network was trained by initially selecting small random values ranging from -0.01 to 0.01 for the weights and off-set values. This was done to start the search in a relatively safe position. A training set of 100 data vectors was then cyclically presented to the network for the process of calculating the gradient and adjusting the weights until one of two termination conditions were satisfied. The first terminating criterion was when the system error $J a$, where

$$
J a=\frac{1}{100} \sum_{i=1}^{100} c_{f_{i}}
$$

was reduced to a value of $\varepsilon$, where $\varepsilon \leq 0.001$. The second terminating criterion was when the number of iterations (i.e., the number of times the training set was cycled through the algorithm) equaled 2000 . The process of computing the gradient and adjusting the weights was performed for each data vector using an iterative process. This process propagated error terms from nodes in the output layer to nodes in the hidden layer, hence the name back-propagation. 
The input weights $w_{i j}$ and output weights $w_{i j}^{\prime}$ were adjusted by the following equations

$$
w_{i j}(t+1)=w_{i j}(t)+\eta \delta_{h_{j}} x_{j}+\alpha\left(w_{i j}(t)-w_{i j}(t-1)\right)
$$

and

$$
w_{i j}^{\prime}(t+1)=w_{i j}^{\prime}(t)+\eta \delta_{j} x_{j}^{\prime}+\alpha\left(w_{i j}^{\prime}(t)-w_{i j}^{\prime}(t-1)\right)
$$

For our classification problem, the gain term $\eta$ was set equal to 0.3 and the momentum term $\alpha$ was set equal to 0.7 . The momentum term was used to make the current search direction an exponentially weighted average of past directions and helped keep the weights moving across flat portions of the search space after they had descended from steep portions (Hush and Horne, 1993). The error terms for the output nodes and the hidden layer nodes were calculated by

$$
\delta_{j}=y_{j}\left(1-y_{j}\right)\left(d_{j}-y_{j}\right)
$$

and

$$
\delta_{h_{j}}=x_{j}^{\prime}\left(1-x_{j}^{\prime}\right) \sum_{k=0}^{3} \delta_{k} w_{j k}^{\prime}
$$

respectively. Furthermore, the internal off-set values for the output nodes $\theta_{k}^{\prime}$ and input nodes $\theta_{j}$ were adapted similarly to the input and output weights as follows

and

$$
\theta_{k}^{\prime}(t+1)=\theta_{k}^{\prime}(t)+\eta \delta_{k} y_{k}+\alpha\left(\theta_{k}^{\prime}(t)-\theta_{k}^{\prime}(t-1)\right)
$$

$$
\theta_{j}(t+1)=\theta_{j}(t)+\eta\left(\dot{x_{j}}\right)^{2}\left(1-\dot{x_{j}}\right)\left(\sum_{k=0}^{3} \theta_{k}^{\prime}(t+1) \delta_{k}\right) \alpha\left(\theta_{j}(t)-\theta_{j}(t-1)\right)
$$

\section{GENESIS Genetic Algorithm}

The GA simulation selected for this study was the GENEtic Search Implementation System, referred to as GENESIS (Grefenstette, 1990). GENESIS is an iterative procedure which maintains a population of candidate solutions to a specified problem. The population members or chromosomes are composed of a string of genes. The population is generated through a series of iterations called generations which consists of the following procedures: selection, mutation, crossover, evaluation, and data collection.

GENESIS provides the option of using either a string representation or a floating point representation for the chromosome structures. This allows structures to be viewed as either binary bit strings or vectors of real numbers. With the floating point representation, each gene within the chromosome is defined by a range of values as well as the number of values specified within the range. In order to obtain reasonable length chromosomes. the weights and off-set values associated with each node of the neural network were encoded into the chromosomes as floating point values. This resulted in chromosomes consisting of 199 genes whose values ranged from -163.84 to 163.84 with incremental values of 0.01 . If a string representation had been selected to encode the network parameters, the length of the chromosomes would have been 2985 bits. A 
second reason for using the floating point representation was that the distance between points in the representation space was proportional to distance measurements within the problem space, and vice versa (i.e., the representation space and the measurement space become "isometric". Michalewicz, 1992). For binary string representations, this property does not hold since distance is normally defined by the number of different bit positions (Michalewicz, 1992). Furthermore, the floating point representation allowed for an easy and efficient implementation of the desired mutation and crossover operators.

GENESIS employs a stochastic selection strategy that guarantees an upper and lower bound on each structure's offspring in the search space (Grefenstette, 1990). Based on previous work performed by Montana and Davis, 1989, a population size of 50 individuals was selected and randomly generated. The selection algorithm allocates each chromosome structure from within the starting population a portion of a roulette wheel whose area is proportional to the structure's relative fitness. Spinning the wheel determines the expected number of offspring for each structure. After the offspring is created, they are randomly shuffled and copied into the new population. The expected number of offspring is calculated by taking the ratio of the performance of the structure to the average performance of the entire population (Grefenstette, 1990).

After the new population was selected, each node from within the hidden and output layers of the two-layer perceptron neural network were given a chance of undergoing mutation. This was implemented by computing the interarrival interval between mutations (Grefenstette, 1990)

$$
\text { Next_Mutation }=\left[\frac{\ln \text { Rand }()}{\ln (1.0-\text { Mutation_Rate })}\right] \text {, }
$$

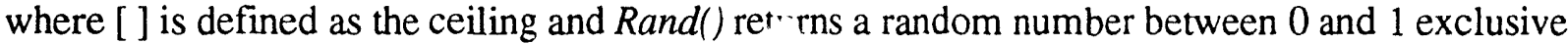
of 1 . For this study, a mutation rate of 50 percent was found to be effective. Once a structure was selected for mutation, one node was randomly selected from either the hidden layer or the output layer of the neural network. The incoming weights and the corresponding off-set value associated with this node were then replaced with random values from a Gaussian distribution with zero mean and unit variance. Care was taken to insure that each randomly generated element fell within the desired range of values specified for each individual gene.

Crossover involved selecting two adjacent parent structures and then copying their respective chromosomes into offspring 1 and offspring2. A crossover point was determined by randomly selecting one node form either the hidden layer or output layer of the neural network and exchanging all respective incoming weights and off-set values between the parents. This process was repeated for every node between the selected node and the final node in the output layer. The two offspring chromosomes were compared outside their crossed areas. If the offspring differed, the segments were swapped. If the offspring do not differ, no crossover is performed and the chromosomes remain unaltered. The offspring were then compared to the parents and if they differed, they were marked for evaluation. Crossover is not performed on all structures within the population. A crossover percentage of 90 percent was chosen prior to initiating the simulation and 
was used in determining the number of structures to be crossed. Thus, only the initial $x$-structures were crossed (Grefenstette, 1990), where $x$ was determined by

$$
x=(\text { Crossover_Rate } * \text { Population_Size }) .
$$

Since the structures were randomly shuffled in the selection procedure choosing only an initial amount of structures resulted in a randomly chosen representative set of chromosomes.

Before evaluation, an elitist strategy was performed that assured the highest paying chromosome would not be lost due to crossover or mutation (Grefenstette, 1990). This procedure searched through the entire population until the highest performing structure was located. Once this structure was located, it was copied into the last position in the new population and was excluded from being changed through crossover or mutation.

After the generation of the new population was completed, individuals were evaluated to obtain a quantitative measure (i.e., fitness) of how will they perform as possible network weights and off-set values. The effectiveness of the individuals were evaluated by assigning each gene to its corresponding node within the two-layer perceptron neural network. Once the weights and offset values were established, the entire test set of 1000 data vectors were fed through the two-layer perceptron neural network and classified. The fitness was then computed as the overall probability of error of assigning each data vector the correct character.

Once the structures had been evaluated, statistics indicating the average performance, the best performance, and the worst performance were calculated. In addition, convergence values were computed to establish whether the population had converged to an individual structure. This was accomplished by determining the number of ones and zeros at each bit position which appear in the string representation for all chromosome structures within the population. When a particular position contains the same value for each structure in the population, that position was said to have converged.

\section{Experimental Results and Conclusions}

Although the overall probability of error of the two-layer perceptron neural network when trained with back-propagation and the GA are comparable, their exhibited behaviors differed considerably. The back-propagation algorithm descended smoothly, rapidly at first, then very gradually. The GA, however, descended in scattered leaps. Training the two-layer perceptron neural network with the GENESIS GA and with back-propagation resulted in probability of errors of $26.1 \%$ and $38.4 \%$, respectively. The back-propagation algorithm obtained this result with 2000 iterations while the GA used 2000 generations each with a population size of 50 , resulting in a total of 100,000 individuals in the search space.

The GENESIS GA has demonstrated an ability to learn the weight set of a two-layer perceptron neural network. The trained network classified data vectors from the test set with a low probability of error. Experimental results indicated that training via the GENESIS GA reduced the 
probability of error of the network to a level slightly worse than that achieved by back-propagation. The weaker performance of the GA is probably due to (1) the size of the search space, i.e.,

$$
\bar{w} \in \Re^{199} \text { and } w_{i} \in[-163.84,163.84]
$$

and (2) the high density of the search space, i.e., the sensitivity of the network to the weights. Thus, a great number of iterations is needed for convergence to an optimal solution and the GA is less likely to successfully find a global minimum without using an inordinate amount of computer resources.

Our hunch is that further study may reveal a better use of the GA algorithm than above. In this extended study we would use the GA to search for an initial weight vector for the neural network, then a standard gradient search technique like back-propagation would be used to find a local minimum near the GA starting point. In this way, both the classical gradient descent technique and the more modern GA method would be utilized in a complementary fashion. This meshing of the two optimization techniques may very well yield results better than either could derive on its own. 


\section{References}

Grefenstette, J. J., A User's Guide to Genesis, Vanderbilt University Department of Computer Science, Nashville, 1984.

Hush, D. R. and Horne, B. G., "Progress in Supervised Neural Networks: What's New Since Lippmann," IEEE Signal Processing Magazine, January 1993, pp. 8-39.

Lippmann, R. P., "An Introduction to Computing with Neural Nets", IEEE ASSP Magazine, April 1987. pp. 4-12.

Marill, T. and Green, D. M., "On the effectiveness of receptors in recognition systems," Trans. IEEE Information Theory, vol. IT-9, 1963, pp. 11-27.

Michalewicz, Z., Genetic Algorithms + Data Structures = Evolution Programs, Springer-Verlag, New York, 1992.

Montana, D. J. and Davis, L., "Training Feedforward Neural Networks Using Genetic Algorithms," Proceedings of the Eleventh International Joint Conference on Artificial Intelligence, vol. 1, 1989, pp. 762-767.

Scherf, A. V. and Voelz, L. D., "Training Neural Networks with Genetic Algorithms for Target Detection," Proceedings of SPIE on Science of Artificial Neural Networks, Orlando, FL, 21-24 April, 1992, vol. 1710, pt. 1:734-741 vol. 2.

Whitley, D., Starkweather, T., and Bogart, C., "Genetic Algorithms and Neural Networks:

Optimizing Connections and Connectivity," Parallel Computing, vol. 14, 1990, pp. 347 361. 


\title{
Genetic Algorithm Based Information Filtering
}

\author{
Kenrick J. Mock \\ Department of Computer Science \\ University of California, at Davis \\ Davis, CA 95616 \\ (916)752-3128,mock@cs.ucdavis.edu
}

\begin{abstract}
As networked systems grow in size, the amount of data available to users has increased dramatically, resulting in information overload. Typically, users face the time consuming task of browsing or searching through much undesirable information. One solution to reduce the search burden placed upon the user is to automatically eliminate incoming data predicted to be irrelevant via an intelligent information filtering system. This paper describes the information filtering problem and compares a hybrid genetic algorithm and hill climbing approach to a global hill climbing approach. Both methods are user modifiable and automatically learn user preferences based upon user interactions with the system. Initial results indicate that this type of real-time problem poses difficulties for standard genetic algorithms. The global hill climbing approach performed slightly better than the genetic approach, achieving up to $70 \%$ accuracy. While achieving less accuracy, the genetic approach appears to be a promising method to increase the breadth of articles retrieved.
\end{abstract}

\section{Keywords: Information Filtering, Genetic Algorithm, Adaptive User Models}

\section{The Information Overload Problem}

This project examined the Usenet News system as a testbed for the filtering algorithm. In the Usenet system, users throughout the world intermittently post articles to a common bulletin board. The number of articles posted may be very large; e.g., newsgroups may receive hundreds of articles daily. The goal is to predict whether new articles are likely to be of interest, or not of interest, based upon the prior behavior of the user. Note that this is an extremely fuzzy problem, as users are notorious for inconsistency and changing interests.

One of the difficult constraints imposed by this type of problem is the necessity for incrementality. Many learning algorithms, e.g. standard neural networks and genetic algorithms, require repeated epochs over a fixed data set. In the news problem, the data set is constantly changing as incoming messages are posted. To ensure consistency, a genetic algorithm or neural network would need to store all messages ever seen; when new messages arrives, the system would retrain on all old examples along with the new examples. This is clearly undesirable due to the time requirements for training and the space required to store all messages. Other approaches to the information filtering problem bypass this problem by typically forcing the user to explicitly define what should be filtered or by using incremental classifiers, such as hill climbing. 


\section{Previous Work}

An example of a keyword/rule-based news filter has been implemented in the Tapestry system (Goldberg et. al, 1992). Tapestry has its own query language, TQL, similar to SQL, which is used to specify what types of information should be retrieved or filtered. Consequently, Tapestry has the power of a full database retrieval system, but is also limited by the vocabulary and keywords which are encoded into the boolean queries. Furthermore, the user must explicitly code the filter using TQL; the system is unable to learn user preferences automatically.

In addition to the keyword approaches, neural networks may also be used to classify incoming articles. Eberts examines the direct approach of feeding raw words into a feedforward backpropagation network (Eberts, 1991). The output units indicate the category of the article, accepted or rejected. Experiments using the header alone and the entire body of the text were conducted. In their tests, the header alone was sufficient to correctly classify articles in most instances. This approach did not address the problem of a dynamically changing data set described in the previous section; it merely tested upon a static store of messages.

A hill climbing approach through competitive agents for information filtering has also been investigated by Baclace (1992) and Stevens (1992). In their approaches, agents are activated depending on terms from the header or body fields of news messages. As the system is used over time, the agents will model the frequency of textual patterns which appear in the articles which are read. Although effective, their systems do not actively explore other messages which may be of interest unless explicitly directed to do so by the user (in the form of reading new messages or creating new agents).

Sheth and Maes (1993) employ a similar strategy as described above, except their system also incorporates a genetic algorithm component to increase the recall rate, or breadth, or articles retrieved. This allows a tradeoff to occur between recall of articles and the precision of the articles which are retrieved. Sheth and Maes allow the user to determine when crossover should occur. At that time, all agents perform a single crossover operation and processing continues. The crossover operation simply swaps words and sensitivity values among agents, and will be discussed in more detail in future sections. The overall result of crossover is lower accuracy, as old agents were destroyed which performed well, but facilitates the retrieval of new newsgroups.

The genetic classification and hill climbing system described in this paper is similar to that proposed by Sheth and Maes, but differs in several regards: (1) Sheth and Maes' scheme operated upon several different newsgroups. This project focuses instead on classification within a single newsgroup, since users typically know which news topics they are interested in. Crossover. Sheth and Maes only perform crossover on newsgroups. The rest of the chromosome remains intact. For example, if the words "genetic" and "algorithm" are of interest in the AI newsgroup, a crossover operation might result in an individual attuned to the words "genetic" and "algorithm" in the Biology or Sports newsgroup. This project performs more aggressive crossover on all extracted features. (3) Automatic Learning. Users were required to initialize individuals in the Sheth and Maes scheme. The approach described in section five creates new individuals automatically. 


\section{Usenet Data}

The data structure for a typical Usenet post is shown below. There are entries for the subject, author, keywords, and other references within the header of each message. Some of these fields are shown below:

Newsgroup: comp.ai

Subject: Genetic algorithms

Author: mock@ucdavis.edu

Body: ... (body of message)

Throughout this project, the only features extracted from each message will be the words in the Subject and Author fields (in order to simplify the scope of the project). For example, in the message shown above, the extracted features would be "genetic", "algorithms", and "mock@ucdavis.edu". Common words such as "the" or "and" are filtered out and are not considered as features. Morphology and spell checking is not performed at this time.

\section{Global Hill Climbing Approach}

All of the previous approaches described in section two rely primarily upon the user to create the filter. A simple method to automate the filtering process is to memorize features extracted from each article and perform hill climbing on those features. As the user reads messages, she indicates whether or not each message read was accepted or rejected. This outcome is used to increment the weights accordingly. An example is shown below, where originally no features or weights exist.

An accepted message vith the features "genetic", "algorithm", and "mock@ucdavis", will result in setting the global table as shown below:

$\begin{array}{lll}\text { Word } & \text { Accepted } & \text { Rejected } \\ \text { genetic } & 1 & 0 \\ \text { algorithm } & 1 & 0 \\ \text { mock@ucdavis } & 1 & 0\end{array}$

Table 1

If the next message is rejected, with the features "dag", "algorithni", and "noelle@suny", the table will be updated to:

$\begin{array}{lll}\text { Word } & \text { Accepted } & \text { Rejected } \\ \text { genetic } & 1 & 0 \\ \text { algorithm } & 1 & 1 \\ \text { mock@ucdavis } & 1 & 0 \\ \text { dag } & 0 & 1 \\ \text { noelle@suny } & 0 & 1\end{array}$

Table 2

Finally, if the next message is accepted with the features "genetic", and "mock@ucdavis", the table will be updated again to: 


$\begin{array}{lll}\text { Word } & \text { Accepted } & \text { Rejected } \\ \text { genetic } & 2 & 0 \\ \text { algorithm } & 1 & 1 \\ \text { mock@ucdavis } & 2 & 0 \\ \text { dag } & 0 & 1 \\ \text { noelle@suny } & 0 & 1\end{array}$

Table 3

In this manner, the algorithm incrementally builds a table which reflects how features have been accepted or rejected in the past, with certain words being key predictors.

Given such a table, classification of new messages is performed by extracting the features from the new article and then computing the sum of all the Accepted and Rejected values from matching features in the table. If the percentage of accepted values exceeds $A$, the message is classified as being of interest. If the percentage is less than $B$, the message is classified as being of no interest. Messages in between are marked unknown. In this project, $A$ was set to 0.7 and $B$ to 0.3 so that the range of percentages could be divided fairly evenly.

\section{Local Genetic Hill Climbing Approach}

The local genetic hill climbing method employed in this project is similar to the global method except instead of a single dynamic table, each individual consists of a set of fixed-sized tables; i.e., each individual performs its own hill climbing. In this project, the table size was set to 20, allowing each individual to identify up to combinations of 20 features. Most messages consist of only 4 or 5 features; consequently each individual has the capacity to represent several different types of messages. These feature tables determine the chromosome of each individual. The entries in each table are initialized to features selected randomly from a set of 100 previously stored messages, and the accepted and rejected values initialized to small random numbers between 0 and 5 . In addition to the table, each chromosome also consists of a history variable which counts the number of times a particular individual has made a correct, or incorrect, prediction. This variable is initialized to zero.

\subsection{Hill Climbing Component}

The classification procedure first finds the single individual who best matches the input. Matching individuals are determined by computing how many features from the input are present in the individual. The individual with the most matching features is selected as most representative of the particular input message. If at least $C$ percent of the features match, then the accepted and rejected values from the matching features of this single individual are added and used to compute a prediction in a manner identical to the that of the global hill climbing approach. In this project, $C$ was set to $50 \%$. If the best individual doesn't match at least $C \%$, it is deemed too distant from the input to make an accurate prediction, and the prediction is set to Unknown.

The hill-climbing learning procedure is outlined as follows. Each new message read by the user is classified as accepted or rejected as described in section four. If rejected, then the rejected values of all features matching the input are incremented in the tables of all individuals. Similarly, 
if accepted, then the accepted values of all features matching the input are incremented in the tables of all individuals. This is a global update similar to the global hill climbing approach.

The next step is to determine the individuals with greater than $C \%$ of its features matching the features of the message. The prediction of all of these individuals is computed; if the prediction equals the actual value, then the history variable is incremented. If it is incorrect, the history is decremented. If the prediction is unknown, no action is taken. This history variable stores the number of times a particular individual has been correct, and will be used in the fitness function.

The final step is to create new individuals if necessary. If the individual with the most matching features has a match percentage greater than $C$, then no additional work is done. The system relies on the initial hill climbing to gradually change the values appropriately. However, if the best individual has a match percentage less than $C$, then no individual exists which matches the input and one must be created. The individual with the smallest fitness and lowest match percentage is selected and its features set to those of the input. If the article was accepted, then all of the new features' accepted values are initialized to positive numbers and the rejected values initialized to zero, and vice versa for rejected articles. In this manner, an individual is modified to exactly match new messages which are far away from all existing individuals. In the process, it may overwrite some old values which are important for classification, but this is hopefully avoided by choosing individuals with a low history value for replacement. Note that this process is important in that it is the only way in which new, applicable features are added into the gene pool.

\subsection{Genetic Algorithm Component}

After the user finishes reading messages, the genetic algorithm component goes into effect. As discussed in section two, it is not practical to store all previously read messages for training. Instead, the system trains in batches, using only the set of messages read in the previous session. With a fitness function based upon these messages alone, it is possible that individuals which classified well on old messages will classify poorly on the new message set. Consequently, it is possible that potentially good individuals which performed well on old messages will be given an unfair fitness. These individuals may then be left out of mating, or may be destroyed during the crossover process.

To help make the fitness more fair to these old individuals, the history variable is included into the fitness calculations. This will bias the fitness by including the previous performance of each individual in the past. For each message in the set of articles read, a prediction is computed using the prediction procedure given in section 5.1. The fitness of each individual is given by the history variable plus the sum of the correct predictions minus the sum of the incorrect predictions.

The crossover operation uses FPR to selects two parent individuals. The actual crossover operation is two point crossover, two indices are selected at random from one of the parents, and all features and values within this selection is switched among parents. The history variables for both parents are then set to the average of the history value of the two parents. Elitism of the top $\mathrm{K}$ individuals is also practiced; the top $\mathrm{K}$ individuals are copied directly into the new population without undergoing any crossover. This project used a value of $\mathrm{K}=3$. 
The mutation operation works by selecting a single individual at random and then either adding a positive or negative random value to one of the accepted or rejected feature values, or by selecting a new feature at random from the set of training messages and replacing a randomly selected feature with the new feature. Other forms of mutation are also possible, for example, replacing words from a thesaurus. The crossover and mutation operations are performed over a pre specified number of generations, as in a standard genetic algorithm.

\section{Comparison of Global Hill Climbing to Local Hill Climbing / Genetic Method}

The global hill climbing method's main strength lies in its simplicity and predictive abilities for features which have been previously encountered. In this case, the system will build a compact representation with no need to worry about destroying potentially good individuals, as is the case in the genetic method. However, the genetic method is actually more powerful than the global method. The global method is unable to discern fine differences in features; e.g., if we are not interested in messages with the features "case-based" and "algorithms" but we are interested in messages with the features "genetic" and "algorithms", then the global method will be using the same accepted and rejected values for the word "algorithms" and may be unable to correctly classify one or both classes of messages. On the other hand, the genetic approach can simply create two separate individuals which can classify both classes of messages. Furthermore, via crossover, the genetic approach will also tend to explore new areas of the search space which would not normally be examined through a strict hill climbing approach. Due to the greater complexity and generality of the genetic approach over the global hill climbing method, training for this scheme should also be more difficult.

\section{Simulation Results}

The global hill climbing algorithm and the local hill climbing genetic algorithm were both tested on a set of 100 messages extracted from the comp.ai artificial intelligence newsgroup. A user read all of these messages and marked each as accepted or rejected. The first 15, 30, and then 50 messages were used for training, and the system attempted to predict the users' choices for the rest of the unread messages. Tests were conducted as though these messages were received in real time. Since approximately 15 messages are posted to this newsgroup each day, the training set was divided up into three sets of 15,15 and 20 messages. The first 15 messages were treated as though they arrived on the first day. These messages were read, and classification was performed on the remaining 85 unread messages to determine the accuracy of the prediction. Then, the next 15 messages were read as though they arrived the next day, and classification was performed on the remaining 70 unread messages. Finally, the next 20 messages were read and classification was performed on the remaining 50 unread messages. Classification accuracy should improve as more messages are read and more information becomes available to the system.

Both the global and local hill climbing and genetic algorithm schemes perform global updates after each message is read. When tested using the genetic algorithm, crossover was performed using the batch of previously read messages; i.e. there are three executions of the genetic component, one using the first 15 messages to determine fitness, the next operating upon the next batch of 15 , and finally the last operating upon the next batch of 20 messages. The 
number of generations varied from 0 to 5 , the probability of mutation was set to $2 \%$, elitism was performed for the top 3 individuals, and the population size was arbitrarily set to 50 since the data set is fairly small (only 100 messages total). Note that although the data set is small, the data space is extremely large, and an experiment with larger data sets to more accurately represent the data space will likely produce more valid results.

\subsection{Global Hill Climbing}

A summary of the results for the global hill climbing method is shown in figure 1 below. As expected, the percentage of correctly classified unread messages increases as more messages are read and more information is known about the interests of the user, ending at $52 \%$ correctly classified after 50 messages are read. The percentage of incorrect messages remains fairly low (including both false positives and false negatives) at about 5\%, while the percentage of unknown messages decreases quickly from a large value to approximately $46 \%$. Global hill climbing ended up giving better overall results than the genetic scheme.

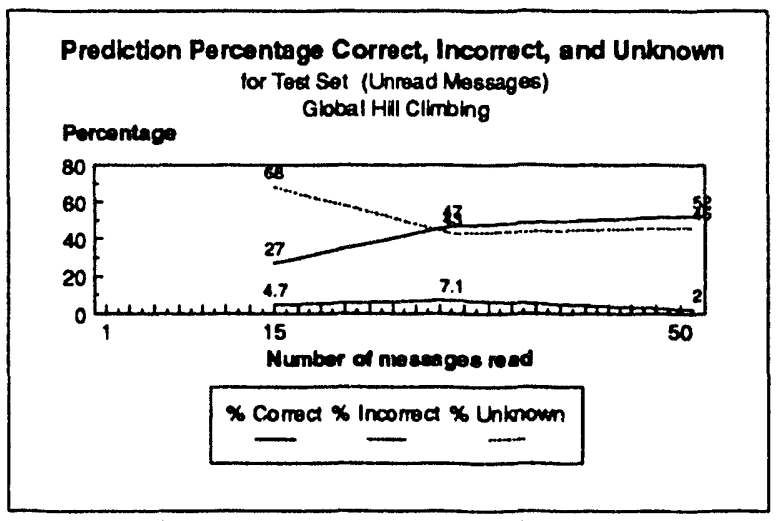

Figure 1. Prediction percentages for Global Hill Climbing Scheme.

\subsection{Local Hill Climbing without Genetic Component}

The local hill climbing method was first examined where each batch of messages underwent 0 generations; i.e., the local hill climbing algorithm operated upon a group of individuals with no crossover or mutation performed at all. The results are shown in figure 2. The percentage of messages correctly classified is less than that of the global method, $42 \%$ opposed to $50 \%$. However, the error rate is equally low and the percentage of unknown messages approximately equal. 


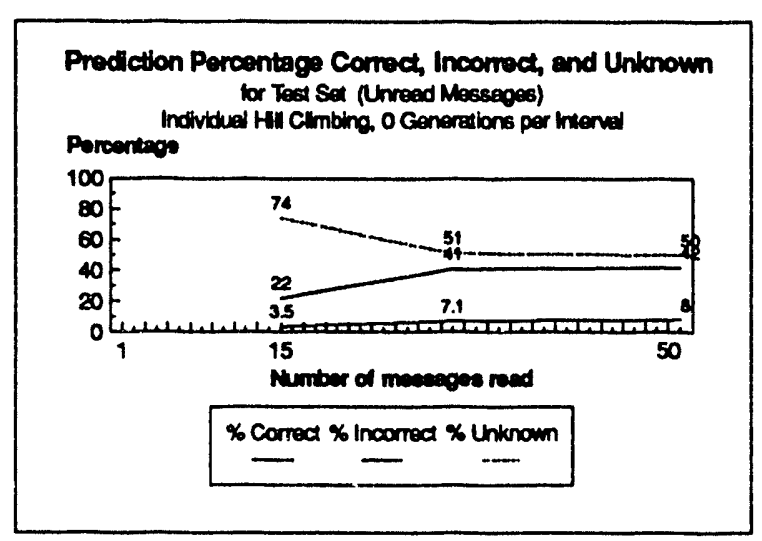

Figure 2. Prediction percentages for Local Hill Climbing / Genetic Scheme, 0 Generations

\subsection{Local Hill Climbing with Genetic Component}

Figure 3 depicts the local hill climbing scheme with a single generation per batch. After the first fifteen messages are processed, a single crossover/mutation operation is performed across all individuals, and then once again after the next fifteen and finally the next twenty messages are read, for a total of three crossover sessions. The percent of correct classifications is slightly higher than the scheme with 0 generations, although the initial error is higher.

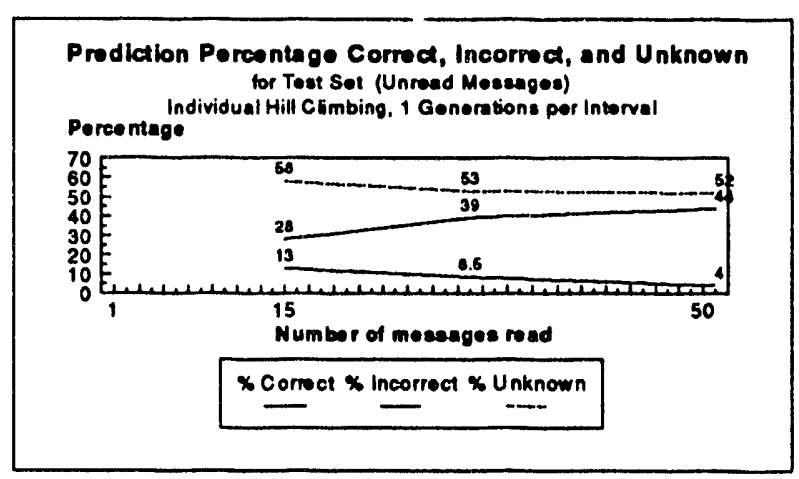

Figure 3. Prediction percentages for Local Hill Climbing / Genetic Scheme, 1 Generations

Figure 4 depicts the prediction percentages for 3 generations per interval. The percent correct matches ( $42 \%$ ) is about the same result as the previous case with 1 generation, although the error is slightly less in this run.

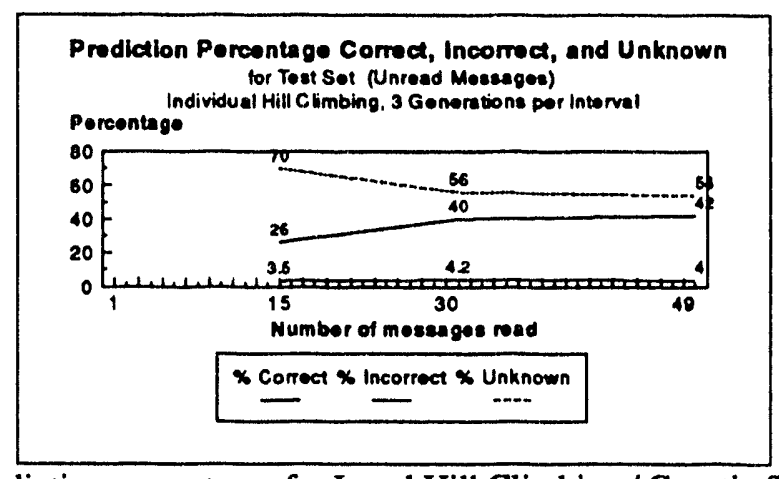

Figure 4. Prediction percentages for Local Hill Climbing / Genetic Scheme, 3 Generations 
A final run with 5 generations per interval is shown in figure 5. At this point, the amount of crossover is destroying too many old individuals which are necessary to perform well, and the accuracy of correct predictions decreases down to $36 \%$. The error stays approximately constant throughout all runs, but the number of unknown messages increases and the percentage of correctly classified messages decreases. Runs with more generations result in worse behavior and are not shown here.

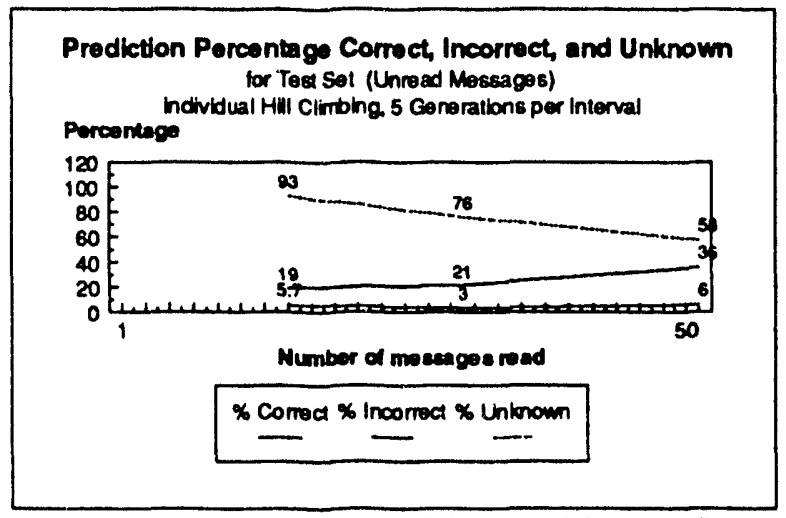

Figure 5. Prediction percentages for Local Hill Climbing / Genetic Scheme, 5 Generations

\subsection{Analysis of Results}

A summary of the results comparing the percentage of correctly predicted messages for the two schemes after 50 messages have been read is shown in figure 6 . The global hill climbing scheme performs best, correctly classifying $52 \%$ of the messages, while the genetic schemes rise to $44 \%$ correct and decrease as more generations are run.

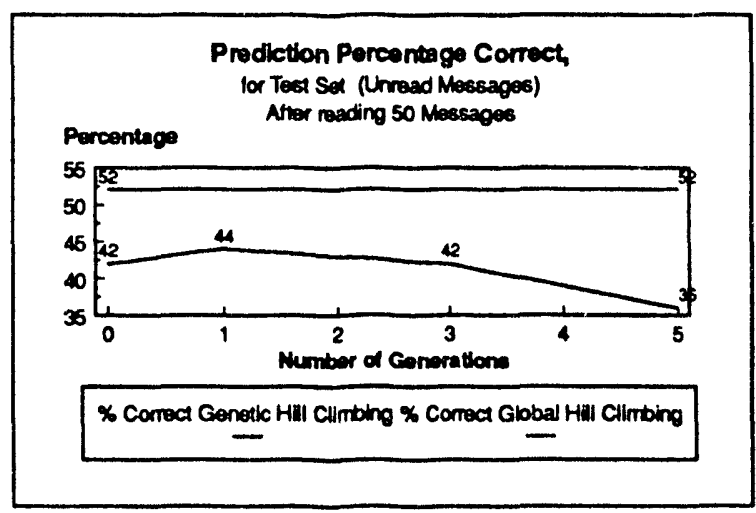

Figure 6. Comparison of Genetic and Global Scheme for various Generations, 50 Messages

While these results indicate that the global scheme performs better on this set of data, further testing is necessary before conclusions may be drawn and generalized. In particular, a larger data set and testing with larger batch sizes may result in significantly different performance. Furthermore, the genetic scheme does perform fairly well on its own, correctly classifying a large portion of the data set and making relatively few errors.

Further insight may also be gained by examining which messages are being classified by the genetic scheme and comparing these to the answers classified by the global scheme. 
Comparison of these answers indicates that the genetic scheme often makes correct classifications of messages which the global scheme marked as unknown. In other words, the genetic scheme has effectively enlarged the search space to include new areas which the global scheme would not consider. In the run with 1 generation per batch, $18 \%$ of the correctly classified answers were classified as unknown by the global scheme. This percentage reached $30 \%$ in the run with 3 generations per batch. Since the genetic scheme is capable of classification in cases where the global scheme is not, the two systems could feasibly be combined together to produce a single system with higher overall precision and breadth than either alone.

\section{Conclusions}

This paper has examined both a hybrid hill climbing/genetic and a strictly global hill climbing approach to the problem of automated information filtering. Both filters adapt to user interests without the need for explicit user programming, and are easily modifiable upon inspection by the user. While both techniques result in fairly good filters, the global hill climbing method appears to give slightly more accurate results. However, the genetic method does appear promising in exploring alternate areas of the data space not examined by hill climbing alone. Further testing with larger data sets, alternate chromosome representations, and examining performance with changing user interests is necessary to better understand the scope and limitations of the approaches presented here.

\section{Acknowledgments}

I would like to thank Ron Braun, Doug Mayfield, R. Borovoy, and Prof. Rao Vemuri for their valuable input and assistance on this and related projects. I would also like to acknowledge Sali and Joon for their conversation and support during many early morning hours.

\section{References}

Baclace, P.E. (1992). "Competitive Agents for Information Filtering," Communications of the $A C M, 35$ (12), 50.

Eberts, R. (1991). "Knowledge Acquisition Using Neural Networks for Intelligent Interface Design," Proceedings of the 1991 IEEE International Conference on Systems, Man, and Cybernetics, 1331-1335.

Goldberg, D., Nichols, D., Oki, B., Terry, D. (1992). "Using Collaborative Filtering to Weave an Information Tapestry," Communications of the ACM, 35 (12), 61-70.

Sheth, B. and Maes, P. (1993). "Evolving Agents For Personalized Information Filtering," Proceedings of the Ninth IEEE Conference on Artificial Intelligence for Applications, 1993.

Stevens, C. (1992). "Automating the Creation of Information Filters," Communications of the $A C M, 35$ (12), 48 . 


\title{
DNA Restriction Fragment Map Assembly with Genetic Algorithms
}

\author{
Walter Cedeño \\ Department of Applied Science and LLNL \\ University of California Davis \\ Lawrence Livermore National Laboratory \\ Livermore, CA 94550 (wcedeno@llnl.gov)
}

\begin{abstract}
The physical mapping of DNA structure for every human chromosome is a task that has occupied many Genome labs for years now. Progress towards this goal can be accelerated with algorithms that are less susceptible to noise in the data and can present the scientist with various optimal or near optimal solutions from the experimental data. Genetic Algorithms have been applied successfully to noisy environments and multi-modal search spaces. This paper describes a Genetic Algorithm to sequence a set of overlapping clones from restriction fragment data using genetic operators suited for multi-modal function optimization. The performance of the algorithm is tested using various data sets. Utility of this approach for mapping parts of human chromosome 19 is described.
\end{abstract}

\section{Introduction}

Mapping the entire human genome is a task that has occupied many Genome labs for years now. The ultimate goal is to obtain the DNA sequence (i.e., order of the bases thymine, cytosine, guanine, and adenine) in every human chromosome. The DNA sequence can be used to identify and locate genes more accurately in the chromosomes. Current technology limits the direct sequencing of DNA to fragments composed of approximately 0.5k base-pairs (Istvanick et al. 1993). In order to divide the DNA into fragments up to this resolution level, techniques using restriction enzymes are used. The restriction enzymes cut the DNA at specific locations which are uniformly distributed in the chromosome. Depending on the number of different restriction enzymes used to obtain the fragments the data is called single-digest (one enzyme), double-digest (two enzymes), or n-digest ( $\mathrm{n}$ enzymes) data. Most mapping is done using single- and double-digest data. Scientists use different restriction enzymes to obtain DNA fragments of the appropriate size.

The Human Genome Center at LLNL is mapping human chromosome 19 which is estimated to be approximately 60 million base-pairs long. The chromosome is divided into islands (contigs ranging between $150 \mathrm{k}$ to $200 \mathrm{k}$ base-pairs) of overlapping cosmid clones, where each cosmid clone is approximately $40 \mathrm{k}$ base-pairs. Each cosmid clone is further divided using a single restriction enzyme (EcoRI) followed by fingerprinting (recording the fragment lengths) into fragments ranging from $0.5 \mathrm{k}$ to $15 \mathrm{k}$ base-pairs. A pictorial view of this process is shown in figure 1 . During the process information about the relative location of the islands, cosmid clones, and fragments in the original DNA is lost. Techniques using larger clones are being used to order, orient, and connect the islands in the original DNA (Olson et al. 1986; Waterman and Griggs 1986; Branscomb et al. 1990; Stallings et al. 1990; Fickett 1993).

The DNA Restriction Fragment Map Assembly problem consists in finding possible locations of the fragments in each clone, and the sequence of the clones in the island so that the fragment overlap is maximized and the total error between the overlapped fragments is minimized. The problem can be pictured in figure 1 as trying to assemble the islands from the fragments and clones. There are other considerations, like the total length of the mapping should be close to the islands original size. The problem is complicated further by the uncertainty in the data and the possibility of data loss (fragments of the same size are hard to distinguish during fingerprinting). In this 
paper we only consider the problem of finding the best clone sequences from the fragment sizes on a set of overlapping cosmid clones.

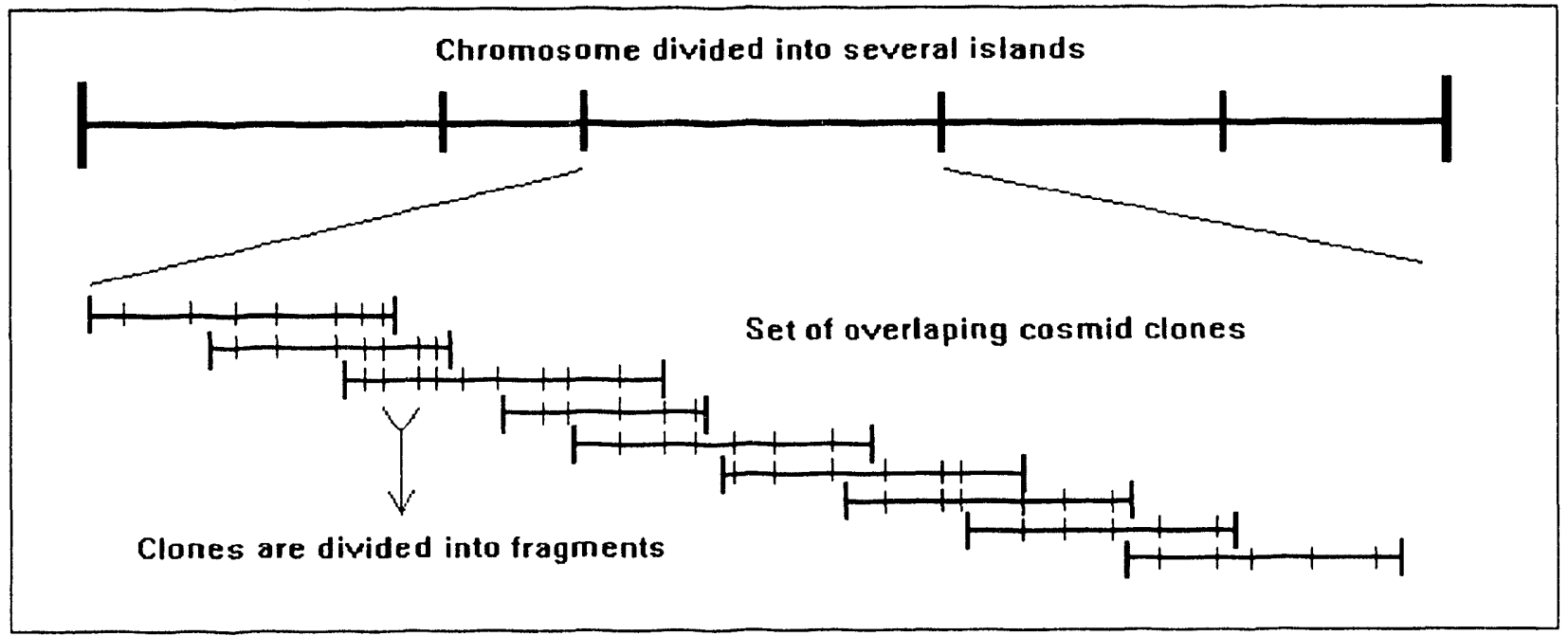

Figure 1: Physical map for island using fragments from a set of overlapping clones.

This problem is known to be NP-hard. For example, the case with 10 cosmid clones, there are 10 ! / 2 possible clone sequences. The problem is complicated further when each cosmid clone contains an average of 9 fragments, whose location within each cosmid clone must be determined. Exhaustive methods for solving this problem are therefore time consuming.

Restriction-site mapping deals with the problem of locating the fragments within clones using restriction digest data. Most techniques being applied to restriction-site mapping make use of single- and double-digest data to determine the location of the fragments within a clone. Stefik (1978) used branch and bound with rules to exhaustively eliminate wrong answers from the digest fragment data. This approach is sensitive to error in the data and is computer intensive. Pearson (1982) exhaustively generated permutations of the single-digest data to compute the error between the generated double-digest and the actual (experimental) double-digest data. This approach is faster but it is limited to small number of restriction sites also. Krawczak (1988) developed a divide and conquer technique that groups the fragments into compatible clusters and then determine the order of the fragments within each cluster. This approach can process a greater number of restriction sites. Platt and Dix (1993) use Genetic Algorithms (GAs) for restriction-site mapping using double digest data. In their work they did not consider operators suited for multi-modal search spaces and mating which preserve adjacency information.

Other techniques are use to sequence larger DNA regions. Branscomb et al. (1990) developed a greedy algorithm to order the most probable clone sequence using overlap probabilities between the clones. The algorithm works well when a large amount of overlap between the clones exists and the fragment data has small errors. This approach is prone to getting stuck in local minima and does not use all the available data gathered at great expense.

Cuticchia et al. (1992) constructed maps using simulated annealing techniques. In their work clones are ordered according to a measure of similarity between them given by the presence or absence of specific sequences. A signature is assigned to each clone and the algorithm uses it to minimize the error between the actual length of the contig and the given length by the hypothetical clone ordering. Matching signatures are use to order the clones. In their work they only considered the relationship between consecutive clones.

This paper describes a GA that uses single-digest restriction data on a set of overlapping clones to find multiple solutions for the clone sequences. The application uses genetic operators suited for multi-modal function optimization (Cedeño \& Vemuri 1992) to determine solutions to the problem from the fragment data sizes. A mating operator based on Genetic Edge Recombination (Whitley et al. 1989) is used to preserve adjacency information and improve convergence towards multiple 
solutions at the same time. Results are given for two data sets of overlapping clones from human chromosome 19.

Section 2 of the paper describes the GA model used for this problem. Section 3 defines the genetic operators for this problem. Section 4 shows the results obtained on different data sets. Section 5 gives comments and conclusions about the approach and section 6 describes future work.

\section{Genetic Algorithms for Multi-modal Search}

Genetic algorithms (Holland 1975) are a search technique based on the principles of natural selection and genetic recombination. GAs have been shown to work well in noisy environments and complex search spaces and have been applied to a wide variety of problems ranging from scheduling to aircraft design (Schaffer 1989; Belew \& Booker 1991). In this section a GA suitable for multimodal function optimization is described.

The GA model used has the ability to converge to multiple solutions at the same time by encouraging competition between individuals within the same local optima. The following pseudocode summarizes the salient features of the method:

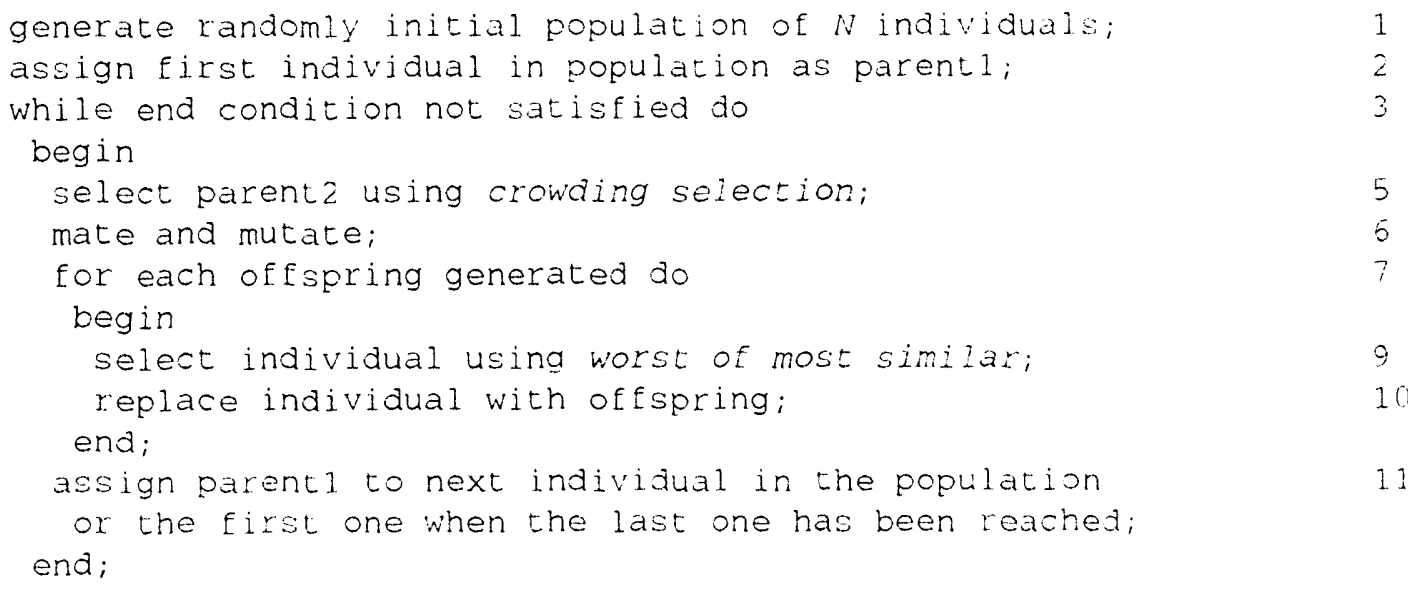

If we use fitness-proportionate reproduction in steps 2,5 , and 11 shown above and replace the lowest fitted individuals in the population (steps 9 and 10) with the newly generated offspring, this model corresponds to a steady-state GA (Whitley 1988; Syswerda 1989). In contrast with the most common generational GA, offspring are available for mating as soon as they are generated, and good individuals can survive for many generations (a generation is every $N$ mating operations in this model).

What makes this model useful in multi-modal search spaces is the use of crowding selection and worst of most similar replacement. In crowding selection a mate is selected as the most similar individual from a group of $C S$ individuals chosen at random from the population. Crowding was introduced by De Jong (1975) where offspring replaced similar individuals from the population. In this model crowding selection is used to promote mating between individuals within the same local minima. In worst of most similar replacement (see figure 2), for each offspring, $C F$ (crowding factor) groups with crowding size individuals selected at random from the population are formed. The individual with the lowest fitness out of the most similar representative in each group is chosen to die. A similar technique called enhanced crowding (Goldberg 1989) has been used before, but there the most similar individual out of a group of worst candidates is replaced.

A function determines the similarity between two individuals by measuring proximity in the decoded parameter space (phenotype). The smaller the value, the closest are the two solutions for the problem. An example of such a metric is the Euclidean distance for function optimization. Measuring distance between decoded genes instead of the genes themselves has been shown (Goldberg \& Richardson 1987; Deb 1989) to give better results for multi-modal function optimization. An example of the use of this function during replacement is shown figure 2 . 


\begin{tabular}{|c|c|c|c|c|c|}
\hline POPUIAT TON & FITNESS & CF Groues & $\begin{array}{l}\text { Similarity } \\
\text { value }\end{array}$ & $\begin{array}{l}\text { Most Similar } \\
\text { of each group }\end{array}$ & $\begin{array}{l}\text { Individual } \\
\text { Replaced }\end{array}$ \\
\hline 10101010 & 89 & 11112000 & 10 & 11111000 & 10111001 \\
\hline 00010010 & 65 & 00000111 & 20 & & \\
\hline 11111000 & 53 & & & & \\
\hline 00100111 & 45 & 11100011 & 3 & 00011011 & \\
\hline 00000111 & 90 & 00111011 & 5 & & \\
\hline 10111001 & 23 & & & & \\
\hline 11100011 & 12 & 00000111 & 20 & 10111001 & \\
\hline 00011011 & 11 & 10111001 & 24 & & \\
\hline
\end{tabular}

Figure 2: Similarity metric applied during replacement with $C F=3$ and crowding size of 2 .

Other approaches for multi-modal function optimization exists. De Jong (1975) use crowding to improve convergence of the GA in multi-modal functions. In crowding offspring are inserted in the population by replacing the most similar individual from a group selected at random from the population. Crowding slows down premature convergence of the traditional GA and in most cases can find the global optima in a multi-modal search space. On the other hand it does not converge to multiple solutions and after many generations one of the peaks takes over the population.

Goldberg \& Richardson (1987) used the sharing concept (Holland 1975) to divide the population into niches according to a similarity measure between them. In sharing, the individuals fitness is degraded according to the presence of other individuals in the same niche. This approach allows multiple solutions to converge in parallel but it is very computing intensive. Deb (1989) applied mating restriction to sharing methods. Mating restriction only allows individuals within the same niche to mate. The method is too restrictive and the user must have an idea of how many peaks are in the search space.

Mahfoud (1992) introduced deterministic crouding. In this approach all individuals are allowed to mate in every generation and offspring competed with their parents for slots in the population. The two parents are paired with the most similar offspring and the individual with the lowest fitness dies. This approach is very fast and simple, but it assumes that mating will produce offspring near their parents. It is not clear if multiple solutions can be maintained for many generations.

Beasley et al. (1993) applies traditional GAs to multi-modal function optimization by using a fitness derating function to prevent convergence to a known local optima. In their approach the GA is applied iteratively to the problem and every solution found in previous iterations is used to derated the fitness of individuals near them. The time complexity is similar to that of sharing functions. Like in sharing the user must have an idea of the search space before hand. Derating a large region may eliminate a possible solution.

The approach used here has been successful in maintaining multiple solutions for function optimization and combinatorial problems (Cedeño 1992). The idea is to maintain diversity by encouraging competition between members from the same niche. To improve convergence lowest fitted individuals are likely to be replaced by offspring belunging to the same niche. No restriction is imposed during mating to allow the exploration of other regions of the space. In the same manner an offspring may replace an individual from another niche, thus allowing competition within niches as well. Survival of the fittest is applied during the replacement step allowing the GA to converge to better solutions within each niche. No prior knowledge of the search space is needed and niches get formed naturally.

\section{Problem Representation}

In this section the problem's dependent genetic operators are defined. First, we will examine the encoding for the chromosome to describe how clone sequences are represented. Second, we describe how the fragment sizes are used to define the fitness function for the problem. Third, the edge recombination mating operator is described. And last. the function that measures similarity between two clone sequences is described. 


\begin{tabular}{|c|c|c|}
\hline ALLELE & CLONE & \\
\hline NUMBER & $\mathrm{ID}$ & FRAGMEINT SIZES (k base-pairs) \\
\hline 0 & 5154 & $16.55 \quad 4.4 \quad 1.68 \quad 1.07 \quad 4.81 \quad 8.5$ \\
\hline 1 & 7442 & $0.79 \quad 0.79 \quad 2.6 \quad 4.35 \quad 8.24 \quad 2.7 \quad 6.9 \quad 5.16$ \\
\hline 2 & 21230 & $\begin{array}{lllllllll}0.96 & 1.68 & 1.08 & 4.77 & 8.47 & 1.44 & 2.37 & 6.29 & 0.62\end{array}$ \\
\hline 3 & 8131 & $0.92 \quad 3.73 \quad 19.8 \quad 4.43 \quad 1.69 \quad 1.25 \quad 4.68 \quad 5.03$ \\
\hline 4 & 18993 & $\begin{array}{lllllllllll}0.96 & 6.31 & 5.48 & 8.61 & 7.29 & 0.81 & 0.81 & 2.6 & 4.36 & 1.92\end{array}$ \\
\hline 5 & 5435 & $2.89 \quad 8.24 \quad 2.7 \quad 6.9 \quad 5.14 \quad 5.14 \quad 2.89 \quad 1.54$ \\
\hline 6 & 7255 & $\begin{array}{lllllllllll}1.04 & 8.21 & 2.69 & 6.89 & 5.12 & 5.12 & 2.88 & 1.94 & 2.42 & 1.37 & 3.33\end{array}$ \\
\hline 7 & 12282 & $4.52 \quad 5.13 \quad 5.13 \quad 2.87 \quad 1.94 \quad 2.42 \quad 1.39 \quad 3.35 \quad 5.41$ \\
\hline 8 & 27714 & $\begin{array}{lllllllllllllll}0.69 & 5.07 & 5.41 & 2.88 & 1.92 & 2.32 & 1.4 & 3.35 & 5.46 & 17.65 & 1 & 10.49 \\
0.58 & 1.74\end{array}$ \\
\hline 9 & 10406 & $2.03 \quad 1.43 \quad 2.34 \quad 6.28 \quad 5.46 \quad 8.58 \quad 7.27$ \\
\hline
\end{tabular}

Figure 3: Cosmid clones with fragment data.

Before going into the details about the operators, it is important to show how the data for the problem is presented to the GA. Figure 3 shows fragment sizes obtained from fingerprinting for a set of overlapping cosmid clones. Some of the fragments from different clones have similar sizes since some of them come from the same original DNA and are obtained using a single restriction enzyme. The data for this problem consist of the $M$ cosmid clones with their fragments and the tolerance measure $E$ which is use to determine if two fragments are of the same size. That is, two fragments $F 1$ and $F 2$ are considered to be of the same size if $|F 1-F 2|<E$.

\subsection{Chromosome Encoding}

The encoding for this problem is very simple. Each allele in the chromosome has a value between 0 and $M-1$ corresponding to one of the cosmid clones. No two alleles have the same value and mating and mutation will preserve this constraint. Using the data in figure 3 , an allele with value 0 corresponds to clone with ID 5154 and an allele with value 9 to clone ID 10406 . The clone sequence $(5154,21230,10406,7255,12282,27714,8131,18993,7442,5435)$ is represented by the chromosome $(0296783415)$. The initial population is generated by picking at random values between 0 and $M-1$ without replacement for each individual.

\begin{tabular}{|c|c|c|c|c|c|c|c|c|c|c|c|c|c|c|c|c|c|c|c|c|}
\hline CLOUE & $\begin{array}{c}\text { Num! } \\
\text { co }\end{array}$ & & $\begin{array}{l}\text { of } \\
c \text {. }\end{array}$ & $\begin{array}{l}\mathrm{ma} \\
\mathrm{c} 3\end{array}$ & $\begin{array}{r}\mathrm{Ech} \\
\mathrm{CA}\end{array}$ & & $\begin{array}{l}\text { oet: } \\
\text { ct }\end{array}$ & $\begin{array}{c}\text { yeen } \\
C 7\end{array}$ & $\begin{array}{c}n \subset 1 \\
C E \\
\end{array}$ & $\begin{array}{l}\text { ones } \\
\mathrm{co}\end{array}$ & $\mathrm{co}$ & & $\begin{array}{l}\text { rote } \\
\text { C? }\end{array}$ & C3 & $\begin{array}{c}\text { erro } \\
04 \\
\end{array}$ & $\begin{array}{l}1 \\
05\end{array}$ & $\begin{array}{l}\text { in } \\
\text { so }\end{array}$ & $\begin{array}{r}\text { the } \\
i 7 \\
\end{array}$ & $\begin{array}{l}\text { mat } \\
c e\end{array}$ & $\begin{array}{l}\text { ches } \\
\text { ce }\end{array}$ \\
\hline $5: 54:$ & 6 & 1 & 4 & 2 & 1 & 0 & 1 & 0 & 2 & 1 & 0 & 5 & 8 & 4 & 4 & 0 & 3 & 0 & 13 & 8 \\
\hline $7442:$ & 1 & 8 & 0 & 1 & 4 & 4 & 4 & 1 & 1 & 0 & 5 & 0 & 0 & 8 & 5 & 12 & 17 & 3 & 9 & 0 \\
\hline $21250:$ & 4 & () & 9 & 3 & $\hat{2}$ & 1 & 3 & 2 & 5 & 3 & 8 & 0 & 0 & 14 & $z$ & 10 & 20 & 10 & 23 & 5 \\
\hline $8131:$ & 2 & 1 & 氵 & 8 & 2 & 0 & 0 & 1 & 2 & i) & 4 & 8 & 14 & 0 & 11 & 0 & 0 & 9 & 13 & 0 \\
\hline $18993:$ & 1 & 4 & ¿ & 2 & 10 & 1 & 3 & $\hat{2}$ & 3 & 4 & 4 & 5 & 2 & 11 & 0 & 10 & 10 & 9 & 6 & 10 \\
\hline $5435:$ & 0 & 4 & 1 & 0 & i & 8 & 6 & 3 & $z$ & 0 & 0 & 2 & 10 & 0 & 10 & 0 & \pm 6 & 4 & 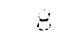 & 0 \\
\hline $7255:$ & 1 & 4 & 三 & 0 & 3 & $b$ & 11 & 7 & 7 & 3 & 3 & 9 & 16 & 0 & 19 & 10 & 0 & 7 & 26 & 23 \\
\hline $12282:$ & 0 & 1 & 2 & 1 & 2 & 3 & 7 & 9 & 7 & 4 & u & 3 & 10 & 9 & $\theta$ & 4 & ; & 0 & 20 & 26 \\
\hline $27714:$ & 2 & 1 & 5 & 2 & 3 & 2 & 7 & 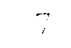 & 14 & 3 & 13 & 9 & 23 & 13 & $1:$ & e & 26 & 20 & ( & 10 \\
\hline 10406: & 1 & 0 & $\Xi$ & 0 & 4 & 0 & 3 & 4 & 3 & 7 & 8 & 0 & 5 & 0 & 10 & 0 & 23 & 20 & 5 & 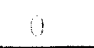 \\
\hline
\end{tabular}

Figure 4: Matrices with the fragment matches and total error between any two clones $(E=10)$.

\subsection{Fitness Function}

To calculate the fitness of an individual the number of fragment matches and the error between the fragments is considered between all consecutive clones. The fragment sizes are represented using integer numbers by multiplying them by 100. All values are computed using integers to accelerate computation of the fitness function. Prior to the execution of the GA two matrices are calculated; one containing the number of fragments that match within tolerance $E$ between any two clones, the other containing the total error between those fragments that match. Figure 4 shows both matrices for the data in figure 3 . The error between two clones is given by the sum of the error between all fragments that matched. For example, between clone 8131 and 5154 there are two 
pairs of fragments, 169 with 168 and 443 with 440 that match within tolerance. The total error between both pairs of fragments is 4 which is the value in the matrix.

From the matrices we can observe that by only looking at the number of fragment matches between the clones it is not sufficient to determine which two clones go together in the sequence. For example, the matches for clone 5154 indicate that clone 21230 (4 matches) must be one of the clones near and either clone 8131 or 27714 ( 2 matches) the other one. In the same manner clone 8131 has 21230 with 3 matches and another 3 clones with 2 matches. Using only the number of matches between consecutive clones was not sufficient to discriminate between clones with the same count. The problem is due to false matches between fragments of similar sizes by chance. By incorporating the total error in the matches the GA is able to discriminate further between clones. Using the same example, clone 8131 has less error in the matches with 5154 than any other clone and therefore indicates that the fragments are more similar. The detail information of the use of these matrices to calculate fitness is given by the following equation:

$$
\sum_{i=0}^{M-1}\left(\frac{\operatorname{Match}\left[C_{i}, C_{l}\right]}{\operatorname{Count}\left[C_{i}\right]} \times\left(1-\frac{\operatorname{Error}\left[C_{i}, C_{l}\right]}{\operatorname{Match}\left[C_{i}, C_{l}\right] \times E}\right)+\frac{\operatorname{Match}\left[C_{i}, C_{r}\right]}{\operatorname{Count}\left[C_{i}\right]} \times\left(1-\frac{\operatorname{Error}\left[C_{i}, C_{r}\right]}{\operatorname{Match}\left[C_{i}, C_{r}\right] \times E}\right)\right) * 100
$$

where $C_{l}$ and $C_{r}$ are the clones to the left and right(if any) respectively of clone $C_{i}$.

From the fitness function note that not only the number of fragment matches are used, but also the percentage relative to the number of fragments in the clone. The reason for this is because we want to give a higher fitness to those clones that match a higher percentage of their regions. For example, clone 5154 has two matches with clones 8131 and 27714 that have 8 and 14 fragments respectively. A higher value is given when the clone 5154 is near 8131 since $2 / 8$ is a higher percentage than 2/14. Dividing by the number of fragments in the clone pushes clones with a higher percentage of matches closer together. The error is used to reduce this count by the percentage of error in the matched fragments. Higher error values will reduce fitness.

Before settling on this fitness function, others were consider; adding the number of matches alone and/or subtracting the error were used. In those cases, the GA was not able to find the correct solution, although parts of the solutions were correct. In future work the number of matches and error between every three clones can be added to improve the fitness measure.

\subsection{Mating and Mutation Operators}

The mating operator is based on a slight modification to the Genetic Edge Recombination (Whitley et al. 1989). This operator was applied successfully to the Traveling Salesperson Problem (TSP). Like in the TSP the important information in this problem is the adjacent alleles and not the order of the alleles in the chromosome. The idea is to recombine the links (pairs of clones) between two parents such that common links are inherit by the offspring. Figure 5 presents an example of this operator. First, those links that are common between both parents are passed to the offspring. Those alleles not passed to the offspring are randomly assigned to the available positions.

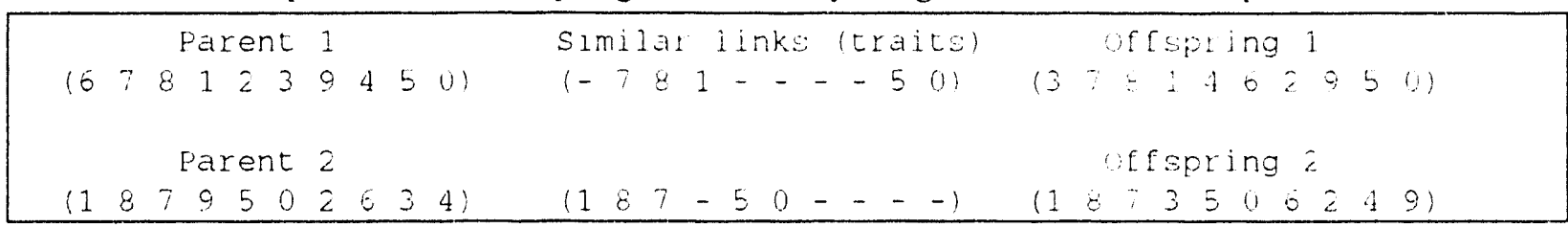

Figure 5: Modified Genetic Edge Recombination for clone sequencing.

The difference between this operator and the original work by Whitley et al. are the number of offspring generated and the assignment of alleles not transferred from the parent. Here we generated two offspring instead of one because the location of the links in the clone sequence is important to our problem. In TSP the chromosome is circular thus the location did not matter. We allow both parents to pass the location of the links to their offspring. To assign the other alleles we select them at random from those clones not passed by their parents. In the original operator the 
links are assigned from those present in any of the two parents. Alleles with fewer links are assigned first to prevent from running out of links for a given allele.

In the mating operator used here there is much more exploration of the search space. This exploration is alleviated by the fact that mates are selected using crowding selection and therefore they have common features between them. Exploration is limited to a smaller region within the entire search space. On the other hand, by allowing clones not assigned to be chosen at random allows links to re-appear that might not have using only mutation.

Mutation is applied on an individual basis. After an offspring is generated it is mutated if a biased coin flip is true. When this happens a link from the offspring is selected at random and all alleles from that link to the last position of the chromosome are reverse. For example, the offspring ( 1873506249 ) after mutation can result in ( 1879426053 ) if the link between allele 7 and 3 is selected to mutate.

The mating and mutation operator are compatible with each other in the sense that they both operate on links. The building blocks of this problems are based on the links between clones in the sequence. The GA operates on these links so that the most useful ones are passed from generation to generation.

\subsection{Similarity Function}

The similarity function is very simple also. It counts the number of dissimilar links between two individuals. Using the parents from figure 5 they have 6 different links corresponding to the five alleles not assigned to the offspring. This metric measures proximity between two clone sequences by the different links they have and not the position of the alleles. For example, the sequence ( 0123456789 ) and ( 9876543210 ) have a distance of zero since all the links are the same. This metric captures the essential aspect of the problem since both solutions are equivalent in our problem.

\section{Results}

The results presented in this section were obtained on a SGI IRIS 4D computer under IRIX O.S. running the GA application written in C. The parameters for the GA are the following:

$\begin{array}{lr}\text { Population size: } & 200 \\ \text { Mutation probability: } & 0.06 \\ \text { Crossover probability: } & 0.95 \\ \text { Crowding selection group size }(C S): & 10 \\ \text { Crowding sub-population size: } & 10 \\ \text { Crowding factor }(C F) \text { : } & 3 \\ \text { Maximum number of generations to execute: } & 100 \\ \text { Tolerance } E \text { : } & 10\end{array}$

These parameters were picked after various tries. The population size of 200 was sufficient for the data submitted to the application. Other sizes (in multiples of 50 ) were tried. but higher sizes did not provide new information about the problem and lower sizes in some cases did not converge to the best solutions seen before in the allowed number of generations. Mutation was set at 0.06 . therefore an average of 12 individuals were mutated every generation. This low value of mutation was selected to avoid eliminating the best of population frequently and allow faster convergence within each niche. In the other hand the mating probability was set to a high value of 0.95 because in our GA all individuals have a higher chance of mating with a similar individual, therefore their similar traits will be passed to the next generation. The group size for crowding selection and crowding sub-population was set at $5 \%$ of the population size with a crowding factor of 3 . For each individual at most $5 \%$ of the population were examined for selection and at most $15 \%$ of the population were examined for replacement. These values allowed a diverse population to co-exist during the number of generations allowed and did not restricted competition between individuals from different niches. The tolerance value $E$ was set to 10 to minimize false matches due to chance. 
Higher values of $E$ increased the false matches more than true matches and therefore more possible clone sequences were found.

Following are some of the best sequences obtained for two different sets of overlapping clones. Set number 1 data is shown in figure 3 and set number 2 data is show. in the appendix. The GA took an average of 50 seconds for each run. Figure 6 shows the actual sequence for the data sets and the clone sequences (with their fitness) obtained by the GA.

Set 1 actual sequence:

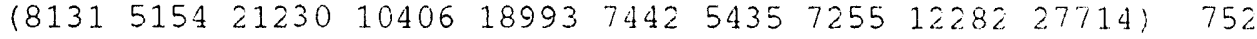

Best sequences found by the GA with fitness:

$\left(\begin{array}{llllllllll}8131 & 5154 & 21230 & 10406 & 18993 & 7442 & 5435 & 7255 & 12282 & 27714\end{array} \quad 752\right.$

$\left(\begin{array}{llllllllll}8131 & 5154 & 21230 & 27714 & 12282 & 7255 & 5435 & 7442 & 18993 & 10406\end{array}\right) \quad 737$

$\left(\begin{array}{llllllllll}8131 & 5154 & 21230 & 10406 & 27714 & 12282 & 7255 & 5435 & 7442 & 18993\end{array}\right) 729$

$\therefore \quad 27714 \quad 122827255 \quad 5435 \quad 7442 \quad 18993 \quad 10406 \quad 21230 \quad 5154) \quad 718$

$\begin{array}{lllllllllll}0131 & 5154 & 21230 & 10406 & 18993 & 27714 & 12282 & 7255 & 5435 & 7442 & 710\end{array}$

Set 2 actual sequence:

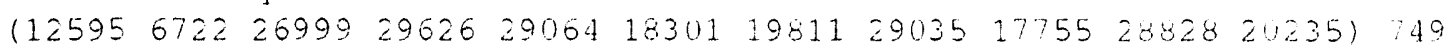

Best sequences found by the GA with fitness:

$\left(\begin{array}{lllllllllll}12595 & 26999 & 6722 & 29626 & 29064 & 18301 & 19811 & 29035 & 17755 & 28828 & 20235\end{array}\right) 75$ :

$\left(\begin{array}{lllllllllll}12595 & 26999 & 6722 & 29626 & 29064 & 18301 & 19811 & 29035 & 17755 & 20235 & 28828\end{array}\right) 755$

$\left(\begin{array}{lllllllllll}12595 & 26999 & 6722 & 29626 & 29064 & 18301 & 28828 & 20235 & 17755 & 29035 & 19811\end{array}\right) 751$

$\left(\begin{array}{lllllllllll}12595 & 26999 & 6722 & 29064 & 29626 & 18201 & 19811 & 29035 & 17755 & 28826 & 20235\end{array}\right) \quad 750$

$(26999 \quad 6722 \quad 12595 \quad 26626 \quad 29064 \quad 18301 \quad 19811 \quad 29035 \quad 17755 \quad 28828 \quad 20235) \quad 750$

$(26999 \quad 6722 \quad 12595 \quad 29626 \quad 29064 \quad 18301 \quad 19811 \quad 29035 \quad 17755 \quad 20235288281748$

$\left(\begin{array}{llllllllllll}12595 & 26999 & 6722 & 29626 & 29064 & 18301 & 28828 & 20235 & 17755 & 19811 & 29035\end{array}\right) 740$

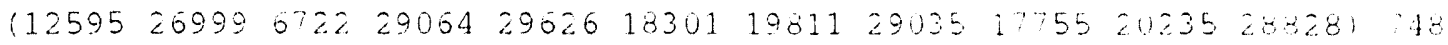

$\left(\begin{array}{lllllllllllll}20999 & 12595 & 0722 & 29026 & 29064 & 18301 & 19811 & 29035 & 175 b & 28328 & 20235\end{array}\right.$

$\begin{array}{llllllllllll}12595 & 26999 & 6722 & 29626 & 29064 & 18301 & 19811 & 17755 & 29035 & 20235 & 28828\end{array} \quad 744$

Figure 6: Clone Sequences obtained by GA and actual Sequence

For data set 1 the GA was able to find the actual clone sequence. From the other sequences found, the fitness values of the next best is 15 less than the actual sequence. Similar gaps exist between all the sequences show in figure 6 for data set 1 . From the solutions we can see that the last four sequences are a single mutation from the actual sequence.

The data for set 2 presented a more challenging problem for the GA. In this case the best sequence found only had clones 6722 and 26999 transposed from the actual sequence. The fitness for the actual sequence is 749 , which is the fifth best score when compared with all solutions found. The actual sequence was obtained in some of the runs, but did not survive until the last generation. Another observation is that there is a difference of 13 or less in the fitness between all the sequences found. Some of the sequences are mutations of others, but there is more diversity when compared with the solutions for data set 1 .

\section{Comments and Conclusions}

Two things were observed that deserved further discussion. Data containing clones with fewer than five fragments were normally sequenced in a wrong location by the GA. This is due to the lack of fragment matches. In a clone the corner fragments will not match with high probability their counterparts in the preceding and succeeding clones. For clones with less than five fragments, it means that in the average, half or more of their region is not useful for fitness and in some cases leads to more false matches. Clone with less than five fragments were usually placed first or last in the clone sequence by the GA. 
When a large amount of overlaps existed between 3 or 4 clones, the GA had difficulty deciding the sequence between them. An example of this behavior was observed with data set 2 . I believe this is happening because the fitness is only looking for the matches between the clones to the left and right separately without accounting for the fragments which are common between all three clones. An improved fitness measure is needed to account for fragment matches between three or more clones.

Overall the GA worked well with the data presented to it. Using the correct set of genetic operators was very important to find a GA model that will find good solutions to the problem. Using a multi-modal approach was very useful for this problem also since it prevented premature convergence and at the same time explored the search space in a more efficient manner. Defining the operators for mating, mutation, fitness, and similarity measure to work with adjacency information between the clones rather than clone positions gave the GA the correct set of tools to converge towards the most probable solutions. More information must be incorporated into the fitness evaluation to distinguish even further between the best clone sequences and other similar ones.

\section{Future Work}

There are other things that I will like to try out in the near future. Improve the fitness function to account for matches between more than two clones. Account for the error between the original island length and the one obtained from the sequence found by the GA and include clone signature information when available. Compare results with other methods available for larger regions. Extend the application to present a partial fragment map using the fragment data. Measure the effect on the GA of different levels of error in the data.

\section{Acknowledgments}

Special thanks to Mr. Tom Slezak and Dr. Elbert Branscomb for describing the problem and providing the test data. Thanks to Dr. Venkateswararao Vemuri for his comments on this work and the preliminary draft of the paper. This work was supported (in part) by the Applied Mathematics Program of the Office of Energy Research (US. department of energy) under contract number W. 7405-Eng-48 to LLNL Lawrence Livermore National Laboratory.

\section{References}

D. Beasley, D. R. Bull, and R. R. Martin. A Sequential Technique for Multi-modal Function Optimization, To be published in Evolutionary Computation, February 1993.

R. K. Belew and L. B. Booker, eds., Proceedings of the Fourth International Conference on Genetic Algorithms, Morgan Kaufmann Publishers San Mateo, CA, July 1991.

E. Branscomb, T. Slezak, R. Pae, D. Galas, A. V. Carrano, and M. Waterman, Optimizing Restriction Fragment Fingerprinting Methuds for Ordering Large Genomic Libraries, Genomics 8, 351-366, 1990

A. J. Cuticchia, J. Arnold, and W. E. Timberlake, The use of simulated annealing in chromosume recunstruction experiment: based on binary scoring, Genetics 132, 591-601, 1992.

W'. Cedeño and V'. Vemuri, Dynamic multi-modal function optimization using genetic algorithms, Proc. of the XVIII Latin. American Informatics Conference, Las Palmas de Gran Canaria, Spain, August 1992.

W. Cedeno, Genetic algorithms in SISAL to solve the file design problem, Proc. of the Second SISAL User's Conference, San Diego CA, December 1992

L. Davis, (ed.), Handbook of Genetic: Algorithms, Van Nostrand Reinhold New York, NY, 1991

K. A. De Jong, An analysis of the hehavior of a class of genetic adaptive systems, Doctoral dissertation. Liniversity of Michigan, Dissertation Abstracts International 36(10), 514013, 1975

K. Deb and D. E. Goldberg, An investigation of niche and species formation in genetic function optimization. Proceedings of the Third International Conference on Genetic Algorithmes, J. D. Schaffer, ed., 42-50, Morgan Kaufmann Publishers San Mateo, CA. June 1989.

J. W. Fickett and M. J. Cinkosky, A genetic algorithm for assembling chromosome physical maps, Unpublished, 1993. 
D. E. Goldberg and J. Richardson, Genetic algorithms with sharing for multimodal function optimization, Proceedings of the Second International Conference on Genetic Algorithms, J. J. Grefenstette, ed., 41-49, Lawrence Erlbaum Associates, Hillsdale, NJ, June 1987.

D. E. Goldberg, Genetic Algorithms in Search, Optimization, and Machine Learning, Addison-Wesley, Reading MA, 1989.

J. H. Holland, Adaptation in Natural and Artificial Systems, University of Michigan Press, Ann Harbor, 1975.

W. Istvanick, A. Kryder, G. Lewandoeski, J. Meidanis, A. Rang, S. Wyman, and D. Joseph, Dynamic methods for fragment assembly in large scale genome sequencing projects, Proceedings of the Twenty Sixth Annual Hawaii International Conference on System Sciences: Architecture and Biotechnology Computing. T. N. Mudge, V. Milutinovic, and L. Hunter eds. IEEE Computer Society Press, 534-543, Wailea, Hawaii, 1993.

M. Krawczak, Algorithms for the restriction-site mapping of DNA molecules. Proc. Natl. Acad. Sci. USA 85, 7298-7301, 1988.

S. W. Mahfoud, Crowding and preselection revisited, Proceedings of Parallel Problem Solving from Nature 2, R. Manner and B. Manderick, eds., 27-36, Elsevier Science Publishers B. V., 1992.

M. V. Olson, J. W. Dutchik, M. Y. Graham, G. M. Brodeur, C. Helms, M. Frank, M. MacCollin, R. Scheinman, \& T. Frank, Random-clone strategy for genomic restriction mapping yeast. Proc. Natl Acad Sci. USA 83, 7826-7830, 1986.

W. Pearson, Automatic construction of restriction site maps, Nucleic Acids Research 10, 217-228, 1982.

M. D. Platt and T. I. Dix, Construction of restriction maps using a genetic algorithm. Proceedings of the Twenty Si.rth Annual Hawaii International Conference on System Sciences: Architecture and Biotechnology Computing, T. N. Mudge, V. Milutinovic, and L. Hunter eds. IEEE Computer Society Press, 756-762, Wailea, Hawaii, 1993.

J. D. Schaffer, ed., Proceedings of the Third International Conference on Genetic Algorithms, Morgan Kaufmann Publishers San Mateo, CA, June 1989.

R. L. Stallings, D. C. Torney, C. E. Hildebrand, J. L. Longmire, L. L. Deaven, J. H. Jett, N. A. Doggett, and R. K. Moyzis, Physic $d$ mapping of human chromosomes by repetitive sequence fingerprinting, Proc. Natl. Acad. Sci. USA 87, 6218-6222, 1990 .

M. Stefik, inferring DNA structures from segmentation data, Artificial Intelligence 11, 85.114, 1978

G. Syswerda, Uniform crossover in genetic algorithms, Proceedings of the Third International Conference on Genetic Algorithms, Morgan Kaufmann Publishers San Mateo, CA, June 1989.

D. Whitley, GENITOR: a different genetic algorithm. Proceedings of the Rocky Mountain Conference on Artificial Intelligence, Denver Colorado, 1988

\section{Appendix}

Fragment data for set 2 of overlapping cosmid clones:

\begin{tabular}{|c|c|}
\hline$\triangle N E=$ & 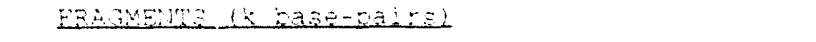 \\
\hline zisten & 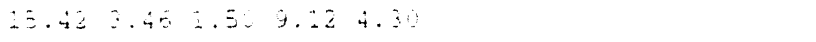 \\
\hline 19811 & 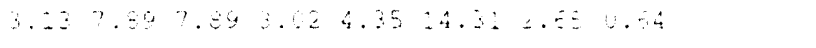 \\
\hline 20996 & 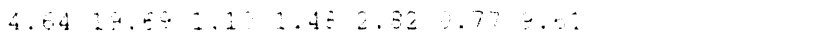 \\
\hline 29620 & $3.4 \div 25 \quad 2.24 \therefore 47 \quad 2349$ \\
\hline 17753 & $1.262 .625 .32 \quad 2.733 .547 .657 .555 .026 .35 \quad 2.74$ \\
\hline 12595 & 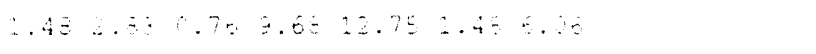 \\
\hline 20235 & 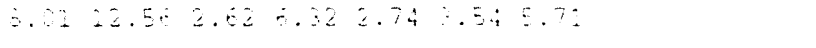 \\
\hline 6722 & 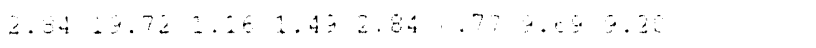 \\
\hline 25825 & $22.452 .62 \quad 0.272 .72 \quad 6.527 .94 \quad 6.30$ \\
\hline 26362 & 2.5312 .952 .5416 .68344 \\
\hline $2=25$ & 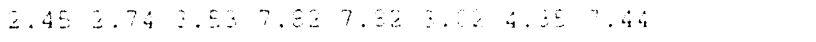 \\
\hline
\end{tabular}




\title{
PROTEIN FOLDING AND GENETIC ALGORITHMS: TORSIONAL ENERGY MINIMIZATION
}

\author{
by Hemant Varma \\ Biomedical Engineering Graduate Group \\ University of California \\ Davis, CA 95616 \\ e-mail:varma@cs.ucdavis.edu
}

\begin{abstract}
The protein folding problem is defined as the prediction of three dimensional structure of a protein from its amino acid sequence. In this paper, no attempt is being made, in any way, to solve this problem, but a very simple problem is considered as a part of the whole process. A very simplified model of the protein is assumed, the backbone dihedral angles are assumed to reach their native conformations for any given protein at minimum torsional potential energy. The torsional potential energy is a function of the dihedral angles phi $(\Phi)$ and psi $(\psi)$ and so at minimum energy, one should be able to find the native conformation values for a given protein. The Genetic Algorithm Routine GENOCOP is utilized and it is found that the above stated hypothesis may not be correct as the overall potential energy for a protein is much more complex, and the possibility of using the GA's for such complex problems is discussed
\end{abstract}




\section{INTRODUCTION}

Biological organisms are made up of thousands of different types of proteins. These proteins are responsible for catalyzing and regulating biochemical reactions, transporting molecules, the chemistry of vision and photosynthetic conversion of light to growth, and they form the basis for structures such as skin, hair and tendons. Protein molecules have remarkable structures. The proteins are basically made of chains of amino acids. This sequence of the chains of amino acids is called the primary structure. Different regions of the sequence form local regular secondary structures such as alpha helices and beta strands. The tertiary structure is the three dimensional structure is formed by folding of these structures to form several compact globular units called domains. It has been reported (Anfinsen,1973) that the information necessary for a protein to fold to its three dimensional or native state is contained within the sequence of the amino acids that form the protein. The protein folding problem therefore is defined as the prediction of the three dimensional structure of proteins from the primary structure or its amino acid sequence. It is one of the fundamental problems in biophysical science. Understanding the physics of protein conformations will be of great importance to biomedicine, in designing novel proteins, in decoding the genetic information obtained by the Human Genome Project, in designing new drugs and in trying to understand the structures and functions of thousands of protein sequences that are being discovered every day in technology laboratories around the world.

One way to approach the problem of protein folding is to go back to the biotechnological laboratories. The three dimensional structure of any given protein sequence can be determined using Xray crystallography or Nuclear Magnetic Resonance (NMR) techniques. But these methods are very expensive and very time consuming. The $\mathrm{X}$-ray crystallography method, for example, currently requires about 3 years of work for each protein studied. And so, the number of proteins for which the tertiary structure is known is a tiny fraction of the number of proteins for which the primary structure is known. In contrast, with the advent of DNA recombinant techniques, now tools are available for the rapid determination of amino acid sequences of proteins from genes. At present, there are about 30,000 (Koza, 1994) proteins with available primary structures and the tertiary structure information is available only for about 400 proteins. Thus it is important that some kind of theoretical prediction methods be formulated.

There have been intensive efforts for the past 25-30 years in theoretical prediction of protein structures from the amino acid sequences. Look at Table I for a summary of the different types of methods that have been formulated so far. Maggiora et al. (1991) have attempted to review these methods in some detail. A good review can also be found in Fasman (1989). Inspite of all the work that has been done, the protein folding problem remains largely unsolved. One of the many reasons for the lack of success is that there are 20 amino acids in nature, and a vast number of ways in which similar three dimensional structures can be generated in proteins by different amino acid sequences. Most of the predictive methods have centered around the prediction of secondary structure of proteins, with the assumption that once one can predict the secondary structure accurately, it might be possible to extrapolate the method to the prediction of tertiary structures. The focus of this paper is on the potential energy based function optimization methods, it must be kept in mind that no attempt is being made here to solve the protein folding problem using this route, only a small problem in the area is being examined. 
Table 1: Prediction methods

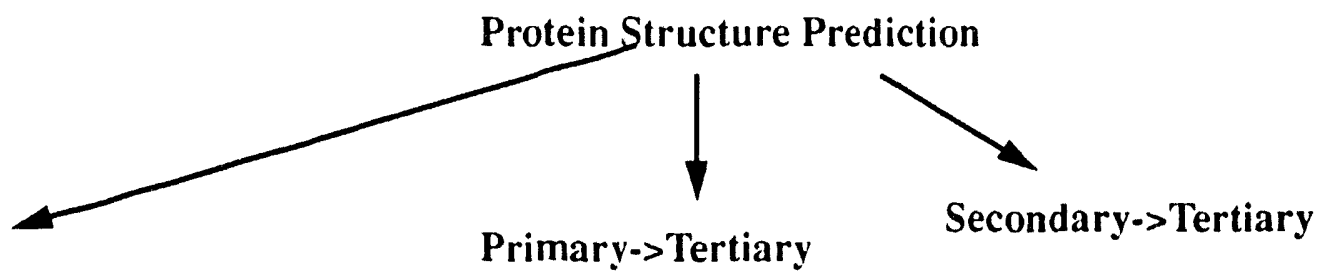

Primary->Secondary

Statistical methods

Pattern based methods

Statistical Mechanical methods

Neural Networks

Energetic methods
????

Homology based methods

Combinatoric Methods

Statistical Methods

Function Optimization

Genetic Algorithms

Neural Networks

Monte Carlo Simulations

Table II: Structural Conformation

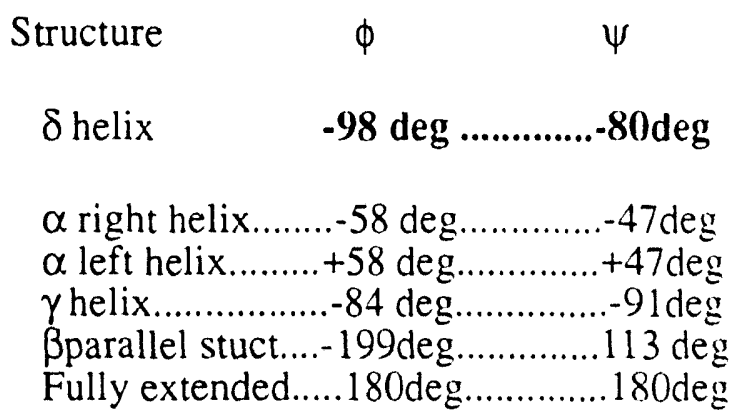

Table III: Results from GA Runs

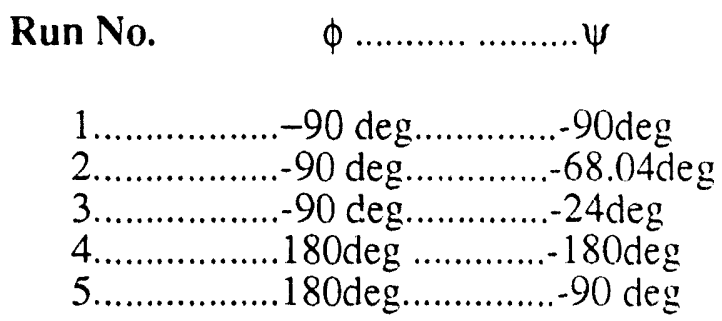


The energy based function optimization methods are broadly based on the concept that a protein in its three dimensional form occupies a minimum energy conformation, and this conformation is considered to be at or near the global minimum of energy. The potential energy functions play a key role in essentially all theoretical studies of proteins and are generally of the form

$$
E=E_{\text {bond }}+E_{\text {ang }}+E_{\text {tor }}+E_{v d w}+E_{h b}+E_{e l}
$$

which is basically a sum of bond energy, angle bending energy, torsional energy, van der Waals forces, hydrogen bonding and electrostatic energy in that order. A good explanation of these terms can be found in any molecular mechanics and molecular dynamics books (McCammon and Harvey, 1987). This potential energy can be minimized as a function of the atomic coordinates to arrive at a local minimum. In this paper I am interested only in the torsional energy as a function of the backbone dihedral angles. In order to explain the problem, discussion follows on the structure of the protein.

\section{THE PROTEIN TORSIONAL ENERGY}

There are 20 amino acids that are found in nature and all proteins are made out of these 20 amino acids. All of these amino acids have in common a central carbon atom $\left(\mathrm{C}_{\alpha}\right)$ to which are attached a hydrogen atom, an amino group $\left(\mathrm{NH}_{2}\right)$ and a carboxyl group (See figure la). The side chain attached to the central carbon atom distinguishes one amino acid from the other. There are 20 different side chains in nature that are specified by the genetic code.

Amino acids are joined end to end during protein synthesis by the formation of peptide bonds. The carboxyl group of the first amino acid condenses with the amino group of the next to eliminate water and yield a peptide bond (See figure $1 b$ ). This process repeats to form a long chain. This chain is referred to as the backbone from which the various side chains project. The polypeptide chain can be divided into peptide units that go from one carbon atom to the next. All the atoms in each such unit are fixed in a plane with the bond lengths and bond angles very nearly the same in all units in all proteins (Branden and Tooze, 1991). Thus the peptide units are effectively rigid groups that are linked into a chain of covalent bonds by the central carbon atoms $\left(\mathrm{C}_{\alpha}\right)$; the only degrees of freedom they have are rotations around these bonds. Each unit can rotate around $\mathrm{C}_{\alpha^{-}}$ $\mathrm{C}^{\prime}$ and $\mathrm{N}-\mathrm{C}_{\alpha}$ (see figure $1 \mathrm{~b}$ ). The angle of rotation around the $\mathrm{C}_{\alpha}-\mathrm{C}^{\prime}$ is called psi $(\psi)$ and the one around $\mathrm{N}-\mathrm{C}_{\alpha}$ is called phi $(\phi)$. Since these are the only degrees of freedom, the conformation of the whole main chain of the polypeptide is completely determined by these two angles of rotation also known as dihedral angles in the case of a helix which is one of the many structures that a sequence of amino acids can fold into. The backbone of the helix is completely defined by the two angles phi and psi and the torsional potential energy is a function of these dihedral angles. The torsional energy of a protein structure is given by

$$
E_{\text {tor }}=K_{\phi}(1+\cos 2 \phi)+K_{\psi}(1+\cos 2 \psi)
$$

here, both the K's are constants relating to some energy barrier and their values differ depending on the protein in question, for our purposes we take a average value of $10 \mathrm{Kcal} / \mathrm{mol}$. Now the hypothesis for this study is that assuming all the other types of potential energies are constant, the 


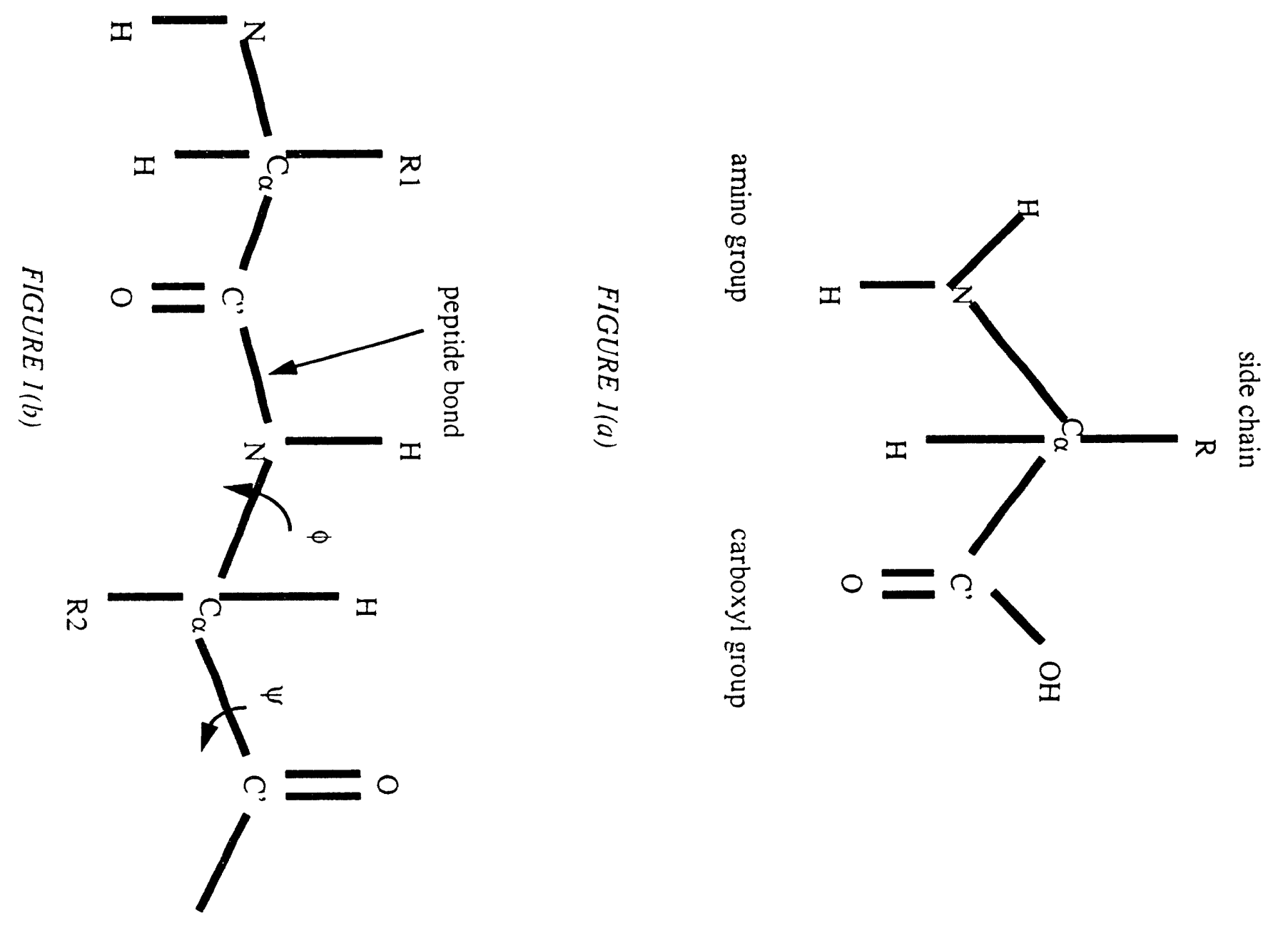


minimization of the torsional potential energy could define the values of phi and psi, the backbone dihedral angles in such a way that the values correspond to some kind of secondary structure.(See Table II for values of phi and psi for corresponding structures). Genetic Algorithms will be used here to minimize this function.

\section{GENETIC ALGORITFIMS}

Genetic Algorithms (Goldbeig, 1989) are search methods based on the mechanics of natural selection and natural genetic operations. They were developed initially by Holland(1975) and his colleagues and have since received increased interest and attention in various fields that closely relate to global optimization.

Genetic Algorithms are a class of machine learning techniques that are similar to certain processes that occur in the interactions of natural, biological genes. A genetic algorithm is a method of finding a good solution to a problem, based on feedback received from its repeated attempts at a solution. The attempts made by a GA are judged by an objective function. each attempt a GA makes toward a solution is called chromosome, which is usually a string of possible solutions.

Typically, a GA will maintain a population of chromosomes. Each chromosome in the population may represent a different sequence and a different idea about how to solve the problem; satisfying the objective function. An objective function must be able to interpret the data contained within a chromosome and decide how good a solution it represents. On applying the objective function once, fitness values are associated with the chromosomes that generate them. Through a sequence of steps, the GA will work towards making its chromosomes more fit. Each pass through a set of optimization steps is called a generation. If all goes well, the overall fitness of the chromosomes will increase with every pass through a generation.

The optimization steps in each generation are known as reproduction, crossover and mutation. In reproduction, chromosomes from the previous generation are duplicated to form a new population. Fitness of a chromosome is assessed by the objective function, and this fitness is the deciding factor in the likelihood of a given chromosome to reproduce. Chromosomes that are more fit are more likely to be duplicated; less fit chromosomes have a poorer chance. Since reproduction is ruled by chance, it is possible that some relatively unfit chromosomes may be allowed to reproduce and the fit chromosomes may not reproduce exactly proportionate to their fitness. It is also important to maintain both fit and unfit chromosomes in the population. The chromosomes that are apparently unfit may contain important information that may be revealed in later generations. The fittest chromosome may be indicating only a locally good solution; such solutions may be less useful in future generations.

After reproduction, the chromosomes undergo crossover. Here pairs of chromosomes are chosen at random and parts of their sequences are exchanged. In performing this step, it is expected that the chromosomes will exchange subsequences that contain good information about solutions and therefore result in chromosomes that are better than their ancestors.

The final step in a generation is mutation. This is executed randomly by altering a very small percentage of the genetic sequences present in the population. Mutation may introduce new concepts 
into the population. This step helps to get a varied population and speed up the search for solutions.

\section{IMPLEMENTING GENETIC ALGORITHMS.}

The genetic Algorithm package, GENOCOP was used to minmize the torsional energy function. The system is based on the article "GENOCOP: A Genetic Algorithm for Numerical Optimization with Linear Constraints" (Michalewicz, in press) and is described in a chapter from Michalewicz(1992). This package is available as a compressed tar file via anonymous FTP from unccsun.uncc.edu (152.15.10.88), directory coe/evol. The authors mention that the current version of the GENOCOP should run without any changes on any BSD-UNIX system, but preferably, Sun Sparkstation. They don't claim the system to have portability. But it is very easy to untar the files and it was very easy to compile the system.

To run the GENOCOP system, there are four simple steps to follow:

1) prepare input file, say, "your_input",

2) incorporate the function in the eval.c file,

3) type "make",

4) type "genocop your_input your_output" ("your_output" is the name of the output file provided by you.).

The speciality of this system is that it can incorporate linear constraints (equalities and inequalities) for the function and the domain of the variables function can be specified, and both of these are part of the information to be provided in the input file. The details on how one can code the input file for optimizing functions with equalities and inequalities as constraint are available in the README file that comes with the package. Here the discussion will be limited to the application being attempted in this paper.

As a first step, the input file is created indicating that the function we are optimizing has two variables and two domains for each of the variables and we also specify that there are no other constraints that we need to take into account. The population size is taken as 70 and 500 generations are allowed in each run. The probability of crossover is taken as 0.65 and mutation 0.01 . There is a variable that needs to be set to 1 for maximizing and 0 for minimizing problem. In this case we need to minimize the function and so that particular variable is set to 0 . The program implements nonuniform mutation and simple crossover. The details of these can be found in Michalewicz(1992).

As the next step a new eval.c file was created with our objective function. The eval.c file for our program would look something like this:

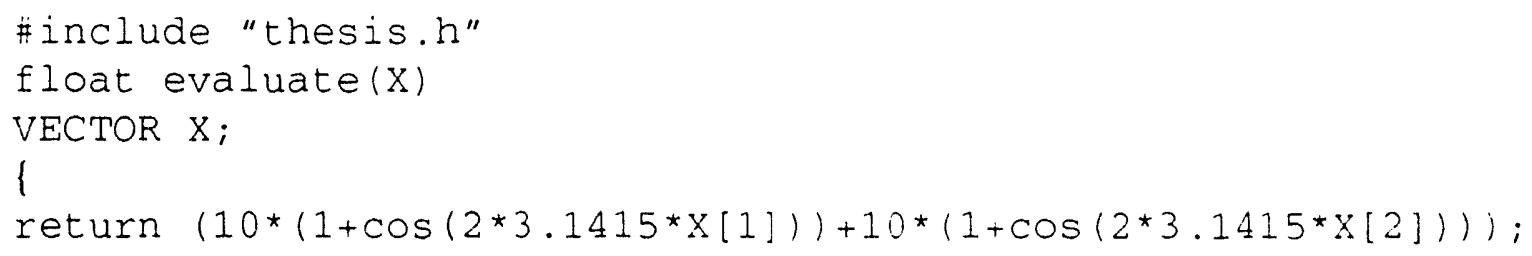


The angles phi and psi have been coverted to $\mathrm{pi}^{*} \mathrm{X} 1$ and $\mathrm{pi} \mathrm{X}^{\mathrm{X}} 2$ so that the $\mathrm{X} 1$ and $\mathrm{X} 2$ are constrained to lie between -1 and 1 and so effectively the two angles are forced to lie between the range -180 and 180 degrees. With the new eval.c file ready, the program is ready to be compiled .

\section{RESULTS AND DISCUSSION}

The results for the values of phi and psi for 5 different runs of the program are listed in table III. Comparing the values of the angles listed in Taule II makes it very clear that the angles for minimum torsional potential energy do not correspond to the structures of any protein as listed in this table II. Therefore it becomes clear that the hypothesis proposed may not be correct. One cannot treat any of the parameters that contribute to potential energy of a protein system in isolation.In order to achieve a accurate prediction of protein conformation one needs to be able to model correctly all the interaction effects at the atomic level, for given amino acid sequence (Sun, 1993).

Schulze-kremer(1992) attempted to use Genetic Algorithms to predict the structure of Crambin. They used a simplified version of the potential energy function taking into consideration only the torsional forces, van der Waals and electrostatic interactions. They also included a term for entropy of the system by defining a pseudo entropic function based on the expected diameters of the atoms. Ideally, I wanted to be able to reproduce the results obtained by Schulze-kremer, for this project, but inability to obtain raw data from PDB was a hindrance. Anyway, the protein used by Schulze was Crambin, and none of the conformations generated were found similar to the native conformation. However, all the conformations generated by the genetic algorithm showed much lower energy than the native structure. This was probably due to the fact that the fitness function used was not a good indicator of the native structure of Crambin.

Sun (1993), probably has achieved the most success in the use of Genetic Algorithms in predicting the tertiary structure based on the potential function. Sun used a reduced representation model to predict the folded structure of melittin, APPI and apancin proteins, from their primary sequences and random starting conformations. The molecular structure of each protein was reduced to its backbone atoms (with ideal fixed bond lengths and valence angle) and each side chain approximated by a single atom. The only parameters allowed to vary were the backbone dihedral angles phi and psi. A potential function which included local and non-local interactions, computed from known structures, was used in structure minimization. With the prirnary structure and the radius of gyration as the only input information, the computed structures were found close to their corresponding crystal structures (three dimensional) for all the three proteins mentioned.

Protein folding studies generally assume the requirement of finding the lowest energy conformation available to the sequence. In fact, it is not known whether the functional conformation of a globular soluble protein is necessarily at the global free energy minimum. The number of possible conformations could be very large, in which case the protein could be considered to follow a sequence encoded pathway from the unfolded to the folded state, which would guide them to the appropriate functional minimum (Levinthal, 1968). Unger and Moult (1993) have shown that protein folding on a lattice model is a member of the class NP-complete problems, and therefore there probably exists no general search algorithm that can be guaranteed to find the global free 
energy minimum for real proteins. The real folding process may thus end up in a functional conformation that is not the global minimum of free energy. As the model becomes more computationally demanding, the general search algorithms are more likely to fail. While the genetic algorithms can be used as general search procedures, they have special properties that are compatible with the folding pathways hypothesis. The genetic algorithms are probably able to mimic the folding pathway rather than conducting a hopeless brute force search for global minimum.

The pathway hypothesis implies that the search algorithms may only be successful if they in some way mimic pathway behavior. Real protein folding pathways have usually been supposed to depend on local regions of chain folding first (early folding units) and the rest of the structure forming around these by a combination of diffusion/collision or propagation process(Wetlaufer, 1973; Karplus and Weaver, 1976; Moult and Unger, 1991). If this is the case Genetic Algorithms may be particularly suitable for reproducing pathway behavior, since they have the property of tending to preserve local favorable conformational features through successive generations. This property is based on the Schema concept (Holland, 1975). A Schema is a pattern used to describe a feature common to many current solutions. Holland has pointed out that short patterns that have above average performance will receive increasing attention during a GA procedure, while below average patterns will be rapidly abandoned. In protein structure applications, Genetic Algorithms may be able to produce a large number of local substructures, concentrate on the favorable ones, and then find the exact way in which these local substructures should be assembled to form a full structure. In this sense genetic algorithms may be considered not as conducting a search on a population of structures, but as sampling points in conformational space of a single molecule along the folding pathway.

\section{REFERENCES}

Anfinsen, C.G.(1973) Principles that Govern the Folding of Protein Chains. Science, 181:223.

Branden, C. and Tooze, J. (1991) Introduction to protein structure. Garland Publishing, Inc. New York and London.

Fasman, G.D.(1989) Prediction of Protein Structure and the Principles of Protein Conformation. Plenum Press. New York, N.Y.

Goldberg, D.E. (1989) Genetic Algorithms in Search, Optimization and Machine Learning. Addison-Wesley, Reading, MA.

Holland, J.H.(1975) Adaptation in Natural and Artificial Systems. The University of Michigan Press, Ann Arbor.

Karplus, M. and Weaver, D.L. (1976) Protein Folding Dynamics. Nature, 260:404-406.

Koza, K. D (1994) Genetic Programming II. Book in progress.

Levinthal, C. (1968) Are there Pathways for Protein Folding?.Jourmal of Chemical Physics, 65:4445. 
Maggiora, G.M., Mao, B., Chou, K.C. and Narsimhan, S.L. (1991) Theoretical and Empirical approaches to Protein Structure Prediction and Analysis. Methods of Biochemical Analysis, Volume 35: Protein Structure Determination 1-87.

McCammon, J.A. and Harvey, S.C. (1987) Dynamics of Proteins and Nucleic Acids. Cambridge University Press, Cambridge, London.

Michalewicz, Z. (1992) Genetic Algorithms + Data Structures= Evolution Programs. Berlin, New York. Springer-Verlag.

Moult, J. and Unger, R (1991) An analysis of Protein Folding Pathways,Biochemistry, 30:38163824.

Schulze-Kremer, S. (1992) Genetic Algorithms for Protein Tertiary Structure Prediction. In:Parallel Problem Solving from Nature 2.eds. R. Manner and B.B. Manderick, Elsevier Science Publication.

Sun, S. (1993) Reduced Representation Model of Protein Structure Prediction: Statistical Potential and Genetic Algorithms.Protein Science, 2:762-785.

Unger, R. and Moult, J. (1993) Genetic Algorithms for Protein Folding Simulations.Journal of Molecular Biology, 231:75-81.

Wetlaufer, D.B. (1973) Nucleation, Rapid Folding and Globular Intra chain Regions in Proteins.Proceedings of the National Academy of Sciences USA, 70:697. 


\title{
The Node Embedding Problem : A genetic algorithm based approach
}

\author{
Dhritiman Banerjee \\ Department of Computer Science \\ University of California \\ Davis, CA 95616 \\ banerjed@lonka.cs.ucdavis.edu \\ Tel : (916) 752-5129
}

December 7, 1993

\begin{abstract}
The node embedding problem deals with the mapping of nodes in a physical network to nodes in an arbitrary virtual topology, so as to establish virtual communication channels between node pairs. The embedding is based on optimization of certain criteria such as minimizing the maximum link flow or the average network delay. The solution is subject to constraints with respect to nodal degree, network-wide wavelength bound and needs to take into consideration the given traffic matrix and the distance matrix between the nodes. The problem has been shown to be NP-hard and hence heuristic based approaches need to be used to model the solution. In this paper we use a genetic algorithm to arrive at solutions to this problem and compare it with results obtained from simulated annealing.
\end{abstract}




\section{Introduction}

Vast improvements in fiber optic technology over the past decade has led to an exciting new era of telecommunications. It has become possible to predict simultaneously servicing thousands of end users, each operating at peak electronic speeds. Wavelength Division Multiplexing (WDM) provides a mechanism to tap into the tremendous bandwidth potential of a single strand of fiber as well as to exploit the other attractive features of optics $[2,4,5]$.

In WDM networks, the fiber bandwidth is carved into orthogonal channels operating at different frequencies (wavelengths), each of which can be used at upto peak electronic speeds. By tuning it's transmitter(s) to one or more wavelength channels, a node can transmit into those channels; similarly, a node can tune its receiver(s) to receive from the appropriate channels. This allows any two stations to set up a virtual channel for communication between themselves irrespective of the underlying physical topology of the network [4].

In a multihop WDM network, virtual communication channels are established between node pairs through proper tuning of the nodal transceivers. Any communication betweer a node and one of its logical (virtual) neighbors can be achieved in a single hop. However, if a node wants to communicate with other nodes, then its message has to "multihop" through intermediate nodes before it finally reaches the destination. If the number of transmitters/receivers (transceivers) per station is high, it becomes possible to communicate with more stations directly and hence fewer packets need to be forwarded by intermediate stations. This reduces the average packet delay, because of the fewer queuing delays at the forwarding intermediate nodes. In particular, if there are $N$ stations and there are $N-1$ transceivers per node, a complete graph can be set up and any station can communicate with every other node directly.

In Figure 1, we show how a $4 \times 2$ torus can be embedded onto a local lightwave network employing a passive star coupler (this approach is also referred to as broadcast and select). A passive star coupler collates all the wavelengths coming in on the input fibers and broadcasts them on the outgoing fibers. Thus each node connected to the passive star coupler can receive the information transmitted by any of the other nodes merely by tuning it's receiver to the correct frequency (similar to tuning a radio to the correct radio station). In the figure, node 0 can directly communicate with nodes 1 and 4 in a single hop (via wavelength channels $\lambda_{0}$ and $\lambda_{8}$, respectively), but if it needs to send a message to node 7 , the message will have to go through intermediate nodes (e.g., from node 0 to node 4 to node 7 ).

The problem of designing a WDM network using a multihop approach can be partitioned into the following subproblems ::

1. Determine the virtual topology ideal for the problem at hand.

2. Map the nodes of the network onto nodes of the virtual topology.

Virtual topologies which have a regular interconnection pattern have proved to be particularly useful in building multihop structures, since they simplify routing (processing at intermediate nodes) to a great extent. In this respect, regular virtual topologies like the hypercube, the Manhattan Street Networks, the ShuffleNet [1] have received considerable attention in the literature. The ShuffleNet is a shuffle-exchange based network which comes very close to achieving the lower bound with respect to average hop distance. The simplified routing schemes in the hypercube make it very useful as a regular virtual topology also. 

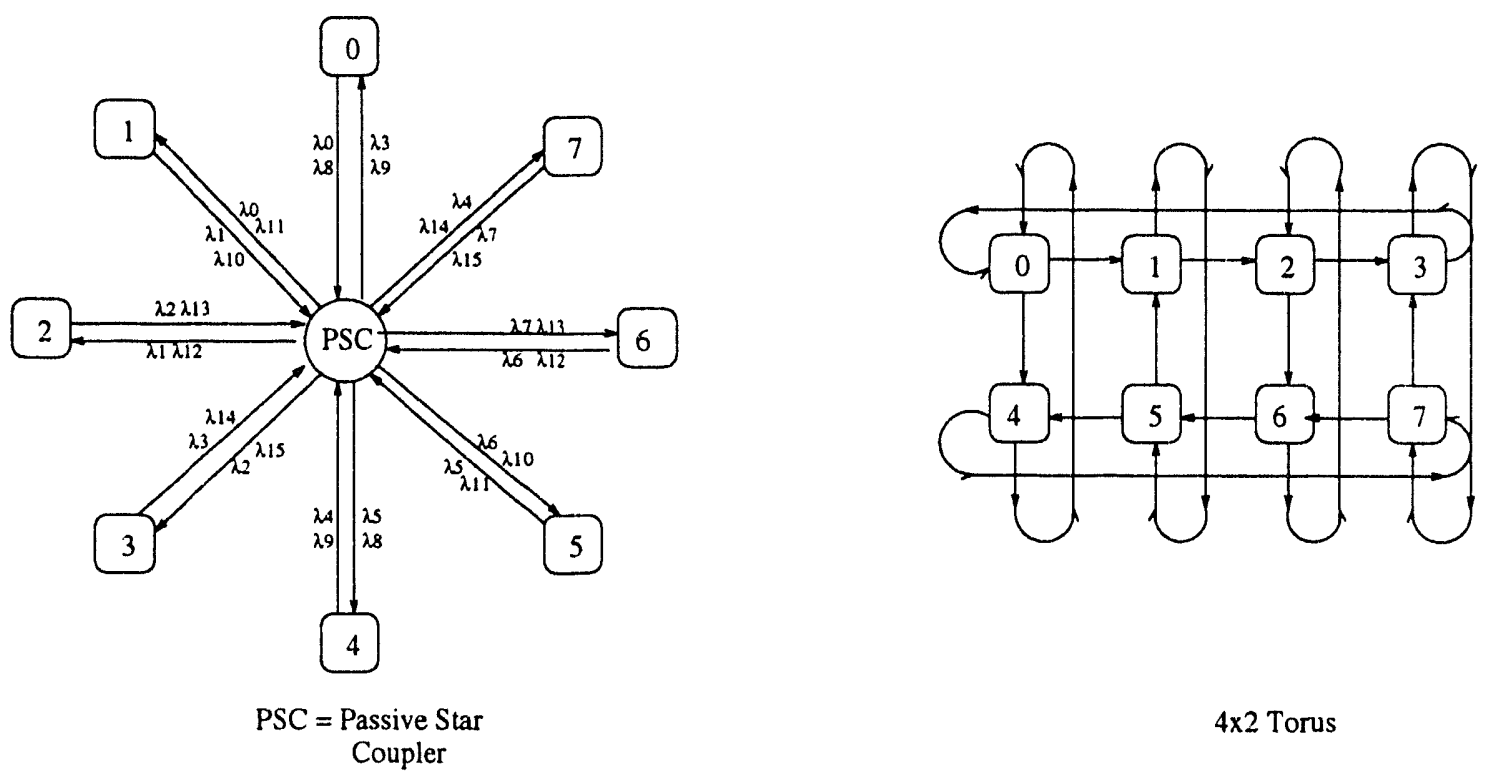

Figure 1: A multihop broadcast-and-select lightwave network embedding a $4 \times 2$ torus.

Once a regular topology has been chosen, the virtual topology design problem reduces to a node mapping problem. The node mapping problem seeks to minimize certain network properties. For example, while minimizing the average delay in the system, one would like to make sure that node pairs which have the maximum traffic between themselves are able to communicate in a single hop. Other optimization criteria include minimizing the maximum flow in a link and load balancing so as to uniformly distribute the traffic in the network links. This allows the overall traffic to scale up as much as possible without any fiber link saturating.

The inputs to the node mapping problem are ::

- The traffic matrix $T$ (where entry $T_{i j}$ denotes the traffic being sent from node $i$ to node $j$ ).

- The distance matrix $D$ (entry $D_{i j}$ denotes the fiber distance from node $i$ to node $j$ ).

- The number of transceivers per node (assumed to be constant across all the nodes, so as to get a regular topology).

This problem has been proved to be NP-hard and there are several heuristic approaches to solving the problem. It has been shown that while a random assignment works well for uniform traffic matrices, substantial gains can be achieved if the traffic matrix is not uniform. If the virtual topology chosen is able to model the traffic pattern correctly then there will be very little forwarded traffic and consequently the average packet delay will reduce.

As a hypothetical case, consider the following situation. Suppose the traffic in a local area network is ring-like in nature, ie. node 1 transmits only to node 2 , node 2 transmits only to node 3 ... node $n-1$ transmits only to $n$ and node $n$ transmits only to node 1 . Then if the virtual topology embedding is a ring [Fig. 2] there will be no forwarded traffic. In the general case given arbitrary traffic patterns, finding an optimal ring embedding would be equivalent to solving the travelling salesman problem.

The virtual topology chosen in our case is a hypercube. The physical topology is arbitrary but it has the same number of nodes as the hypercube. It is also assumed that the optimizing criterion is minimizing the average propagation delay encountered by all packets. 


\begin{tabular}{|c|c|c|c|c|c|c|c|c|}
\hline & & & & & & & & \\
\hline & 0 & 30 & & & & & & \\
\hline & 0 & 0 & & & & 0 & & \\
\hline & 0 & 0 & 0) & 8 & & 0 & & 0 \\
\hline & 0 & 0 & 0 & 0 & & 0 & & 0 \\
\hline & 0 & 0 & 0 & 0 & 0 & 4 & 0 & ( \\
\hline & 0 & 0 & 0 & 0 & & 0 & 6 & ( \\
\hline & 0 & 0 & 0 & 0 & 0 & 0 & 0 & 4 \\
\hline & & 0 & & 0 & & & & \\
\hline
\end{tabular}

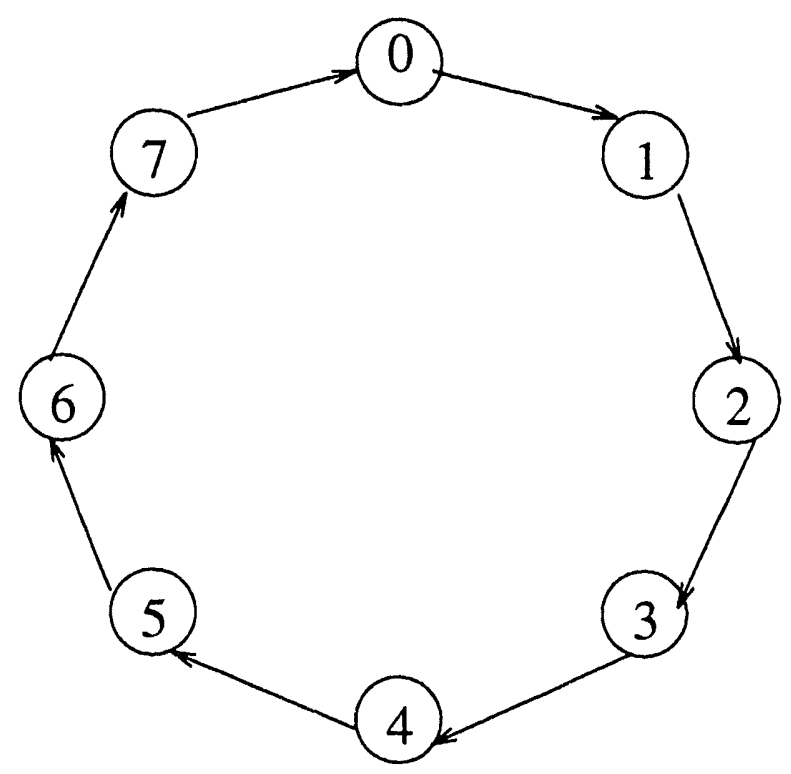

Figure 2: Optimal node placement for a traffic matrix

In this paper we model the problem using a genetic algorithm The results are compared with those obtained by simulated annealing and found to be comparable.

\section{The Genetic Algorithm Approach}

Genetic algorithms have been known to provide good solutions to NP-hard problems, if the problem can be modelled accurately enough. These are search based algorithms which are based on the mechanics of natural selection and natural genetics. They combine survival of the fittest among string structures with a structured yet randomized information exchange to form a search algorithm with some of the innovative flair of human search. Search based algorithms like these have achieved increased popularity as researchers have recognized the shortcomings of calculus-based and enumerative schemes. Genetic algorithms use random choice as a tool in a directed search [6] to arrive at an optimal solution..

In this case, for the node embedding problem, when we are trying to find an optimal solution from the vast number of possible combinations (approximately $N !$ ), it beccmes particularly useful to have randomized algorithms which would not get stuck in a local minima - genetic algorithms perform admirably in this respect, as do simulated annealing techniques which we shall look at in the next section.

For the genetic algorithm approach, the node embedding problem was formulated in the following way :: The virtual topology is assumed to be a hypercube. Each chromozome consists of some random permutation of the numbers from 1 to $\mathrm{N}$, with no duplicates (even though some of the examples in this section assume $\mathrm{N}$ to be 8 , the actual experiment was conducted on 16 nodes). Let us assume that the numbers are arranged in an array $T$. Then $T[i]$ would refer to the physical node which maps to node $i$ in the virtual topology. There would be no duplicates in the $T$ array.

The mutation operation is supposed to find neighbouring configurations in the search space. Thus the mutation operation was performed through a node exchange, in which two adjacent neighbors switch places. 
The crossover operation is used to combine good properties of subsolutions so as to get a better overall solution. In the normal crossover operation, a pair of strings are cut at an arbitrary point and cross-combined. If the parents individually contained desirable properties in different parts of the strings, then the children might inherit good qualities from both parents. However, in our problem formulation, arbitrary crossovers are not permissible. The problem is solved as follows :

eg. Assume that the initial embedding of the parents (assuming 8 nodes) were :

Parent1 :- $\begin{array}{lllllllll} & 4 & 3 & 1 & 7 & 0 & 6 & 5 & 2\end{array}$

Parent2 :- \begin{tabular}{lllllllll}
\hline & 0 & 0 & 1 & 7 & 6 & 2 & 4
\end{tabular}

An ordinary crossover would produce the following children :

Child1:- $\begin{array}{lllllll}4 & 4 & 1 & 7 & 6 & 24\end{array}$

Child2 :- $\begin{array}{llllllll} & 5 & 0 & 7 & 0 & 6 & 5 & 2\end{array}$

Since there are duplicates in the node embeddings this is not an acceptable solution. eg. node 1 occurs twice in Child1 while node 5 does not occur at all. To get an acceptable solution, we would need to make sure that each subpart of a parent embedding are a permutation of the corresponding embedding in it's crossover counterpart. To do this, we do node exchanges in one of the parents. In this case, node 1 and node 0 , node 4 and node 5 are exchanged in Parent 1 so as to get the following configuration before crossover :

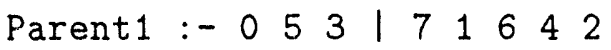

Parent2 :- $\begin{array}{lllllllll} & 5 & 0 & \mid & 1 & 7 & 6 & 2 & 4\end{array}$

A crossover at this point would produce the following

children :

Child1:- $0553 \mid 117624$

Child2 :- $\begin{array}{lllllllll} & 5 & 0 & 1 & 7 & 1 & 6 & 2 & 4\end{array}$

The $\mathrm{C}$ code written by Goldberg was used with slight modifications for the experiments performed. Some of the modifications to the code are listed below :

- The initpop function in the file initial.c is changed to reflect the above coding of the allele. Since we fix the number of nodes in the network to be 16 ie. $N=16$, we do not need to read in the length of the chromozome.

- The objective function needs to be changed so as to call the calcDelay function in embedding.c. However, the code in Goldberg is for finding the maximum in a function. Since we would like to find the configuration with the minimum delay, the fitness value stored is the negative value of the delay calculated. The initial value of the bestfit is also changed to reflect this fact.

- The printout function in report.c was changed to printout the chromozome in a more readable format suitable to the problem at hand. 


\section{Simulated Annealing}

In the simulated annealing process, the algorithm starts with an initial random configuration for the node embedding. Then it goes through a series of node exchange operations so as to reach a better overall average delay for the network. In a node exchange operation adjacent nodes in the virtual topology are switched to get neighbouring configurations eg. if node $i$ is connected to nodes $j, a$ and $b$ and node $j$ is connected to node $p, q$ and $i$ in the virtual topology, after the node exchange operation node $i$ will be connected to $p, q$ and $j$ and node $j$ will be connected to nodes $a, b$ and $i$. Neighbouring configurations which give better results than the current solution are accepted automatically. Solutions which are worse than the current one are accepted with a certain probability which is determined by a system control parameter. The probability with which these failed configurations are chosen, however, decreases as the algorithm progresses in time so as to simulate the "cooling" process associated with annealing. The probability of acceptance is based on a negative exponential factor and is inversely proportional to the difference between the current solution and the best solution so far obtained.

The initial stages of the annealing process looks at random configurations in the search space so as to obtain different initial starting configurations without getting stuck at a local minimum like other greedy approaches. However, as time progresses, the probability of accepting bad solutions goes down, and the algorithm settles down into a minimum after a certain point of time. The state become "frozen" when there is no improvement in the objective function of the solution even after a large number of iterations.

In this particular problem formulation, the propagation delays over the virtual topology are calculated after each adjacent configuration has been obtained. The queuing delays are neglected at this point, since it has been studied that at reasonable loads, the propagation delays are much larger than the queuing delays. Adjacent configurations which achieve lower average delays are accepted, while others are accepted with a certain decreasing probability. The algorithm terminates when a large number of iterations do not fail to improve the solution any further.

\section{$4 \quad$ Numerical Results}

The genetic algorithm as well as the simulated annealing algorithm were both used to map the physical topology of Fig. 3 into a virtual hypercube topology. The traffic matrix employed for this mapping is an actual measurement of the traffic on the T1 NSFNET backbone for a 15-minute period (11:45 pm to midnight) on January 12, 1992. The raw traffic matrix showing traffic flow in mesgs/sec between network nodes was modified to round off the numbers and is shown below. Nodal distances used are the actual geographical distances, and they are not shown here separately.

The 16-node hypercube cannot be directly embedded onto the NSFNET since there are only 14 nodes in the network. Hence the fictitious nodes $A B$ and $X Y$ were added during the initial embedding stage. Since these nodes do not source any traffic, they do not contribute in any way to the delay obtained in the system. However, due to the partial embedding, some of the nodes (eg. Newark, Colorado etc.) have less than 4 transceivers.

An initial approach consisted of a greedy approach in which the nodes were assigned their positions in the reverse order of their nodal degree ie. the station with the highest number of transceivers was assigned it's position first, then the one with the next highest nodal degree and so on. Once the initial embedding had been done, a greedy approach using node exchange was used 


\begin{tabular}{|l|l|l|l|l|l|l|l|l|l|l|l|l|l|l|l|l|l|}
\hline \multicolumn{10}{|c|}{ Raw TrafficMatrix(10000bytes/15min) } \\
\hline & $A B$ & $W A$ & $C 1$ & $C 2$ & $U T$ & $C O$ & $T X$ & NE & IL & $P A$ & $G A$ & $M I$ & $N Y$ & NJ & $M D$ & $X Y$ \\
\hline AB & 0 & 0 & 0 & 0 & 0 & 0 & 0 & 0 & 0 & 0 & 0 & 0 & 0 & 0 & 0 & 0 \\
WA & 0 & 0 & 0 & 0 & 0 & 0 & 0 & 0 & 0 & 0 & 0 & 0 & 0 & 0 & 0 & 0 \\
CA1 & 0 & 0 & 0 & 2 & 0 & 4 & 0 & 1 & 4 & 1 & 4 & 0 & 2 & 0 & 4 & 0 \\
CA2 & 0 & 0 & 0 & 0 & 0 & 0 & 0 & 0 & 0 & 0 & 0 & 0 & 0 & 0 & 0 & 0 \\
UT & 0 & 0 & 0 & 2 & 0 & 0 & 0 & 0 & 1 & 0 & 0 & 1 & 0 & 0 & 0 & 0 \\
CO & 0 & 0 & 4 & 2 & 0 & 0 & 1 & 2 & 5 & 2 & 1 & 0 & 4 & 0 & 1 & 0 \\
TX & 0 & 0 & 0 & 1 & 0 & 1 & 0 & 1 & 0 & 0 & 2 & 0 & 0 & 0 & 1 & 0 \\
NE & 0 & 0 & 1 & 5 & 0 & 2 & 1 & 0 & 14 & 2 & 2 & 0 & 4 & 0 & 1 & 0 \\
IL & 0 & 0 & 4 & 12 & 0 & 5 & 0 & 12 & 0 & 5 & 7 & 1 & 5 & 0 & 2 & 0 \\
PA & 0 & 0 & 3 & 4 & 0 & 4 & 0 & 4 & 10 & 0 & 3 & 1 & 13 & 2 & 0 & 0 \\
GA & 0 & 0 & 5 & 1 & 0 & 2 & 1 & 1 & 9 & 2 & 0 & 1 & 3 & 0 & 0 & 0 \\
MI & 0 & 0 & 0 & 0 & 0 & 0 & 0 & 0 & 0 & 0 & 0 & 0 & 0 & 0 & 0 & 0 \\
NY & 0 & 0 & 5 & 0 & 0 & 3 & 0 & 2 & 6 & 7 & 3 & 0 & 0 & 0 & 0 & 0 \\
NJ & 0 & 0 & 0 & 0 & 0 & 0 & 0 & 0 & 0 & 0 & 0 & 0 & 0 & 0 & 0 & 0 \\
MD & 0 & 0 & 2 & 0 & 1 & 2 & 1 & 1 & 2 & 0 & 0 & 0 & 0 & 0 & 0 & 0 \\
XY & 0 & 0 & 0 & 0 & 0 & 0 & 0 & 0 & 0 & 0 & 0 & 0 & 0 & 0 & 0 & 0 \\
\hline
\end{tabular}

to tweak the existing embedding to get a better solution.

In the reverse-order refinement algorithm, the hypercube embedding as obtained by the initial embedding algorithm was tweaked and the average delay dropped substantially with as few as 10 tweaks. The final solution obtained had an average delay of $10.39 \mathrm{~ms}$ when shortest path routing on the virtual topology was used. Note that this number is quite encouraging compared to the coast-to-coast one-way propagation time of nearly $23 \mathrm{~ms}$.

In the simulated annealing process, the results were even more impressive. The final solution as shown in Fig 4 had a network-wide average delay of $9.715 \mathrm{~ms}$ without considering queuing delays, and $9.720 \mathrm{~ms}$ when queueing was considered (assuming T3 links of $45 \mathrm{MBps}$, and a standard M/M/1 queueing model).

In the genetic algorithm approach, the experiments were conducted over a population size of 50 over 40 generations. Different values were tried for the mutation and the crossover probabilities. Runs with a high probability for mutation and a low probability for crossover were in general found to perform better. The minimum delay was observed to be $10.071 \mathrm{~ms}$ in the case shown in Fig 5 .

\section{Conclusion}

The genetic algorithm approach seems to have done slightly worse than the simulated annealing approach under the present set of data. However, the results are comparable, and given a different set of parameters it might be possible that the genetic algorithm would outperform the simulated annealing process. In general it might be useful to use both approaches in order to arrive at a better solution. Given the fact that the runs with a low probability for crossover tended to do better, it appears that the present crossover method was not amenable to the problem definition. It would be useful to study other ways of performing crossover and mutation operations so as to lower the delay and add an intuitive reasoning to the entire process. Given the hard nature of the problems at hand, it seems that randomized algorithms like Genetic Algorithms and Simulated Annealing 
would perform better than non-heuristic based approaches.

In general, we would expect that the average delay would reduce as the nodal degree is increased. The delay would achieve a minimum if the nodal degree was $N-1$ and the virtual topology was a complete graph so that each node could communicate with every other node in a single hop. This gives us the lower bound for the average packet delay. The average packet delay in this case was computed to be $9.39 \mathrm{~ms}$.

\section{References}

[1] A. S. Acampora, "A Multi-Channel Multihop Local Lightwave Network," Proc., IEEE GLOBECOMM '87, Tokyo, Japan, pp. 37.5.1-9, 1987

[2] C. A. Brackett, "Dense Wavelength Division Multiplexing Networks: Principle and Applications," IEEE Journal on Selected Areas in Communication, vol. 8, no. 6, pp. 948-964, August 1990.

[3] M. G. Hluchyj and M. J. Karol, "Shufflenet: An application of Generalized Perfect Shuffles to Multihop Lightwave Networks," Journal of Lightwave Technology, vol. 9, no. 10, pp. 1386-1397, 1991.

[4] B. Mukherjee, "WDM-Based Local Lightwave Networks - Part II: Multihop Systems," IEEE Network Magazine, vol. 6, no. 4, pp. 20-32, July 1992.

[5] K. N. Sivarajan, "Multihop Logical Topologies for Gigabit Lightwave Networks," IEEE Lightwave Technology Systems, pp. 20-25, August 1992.

[6] David E Goldberg, "GENETIC ALGORITHMS in Search, Optimization, and Machine Learning". 
Banerjee : Virtual Topology Embedding Problem

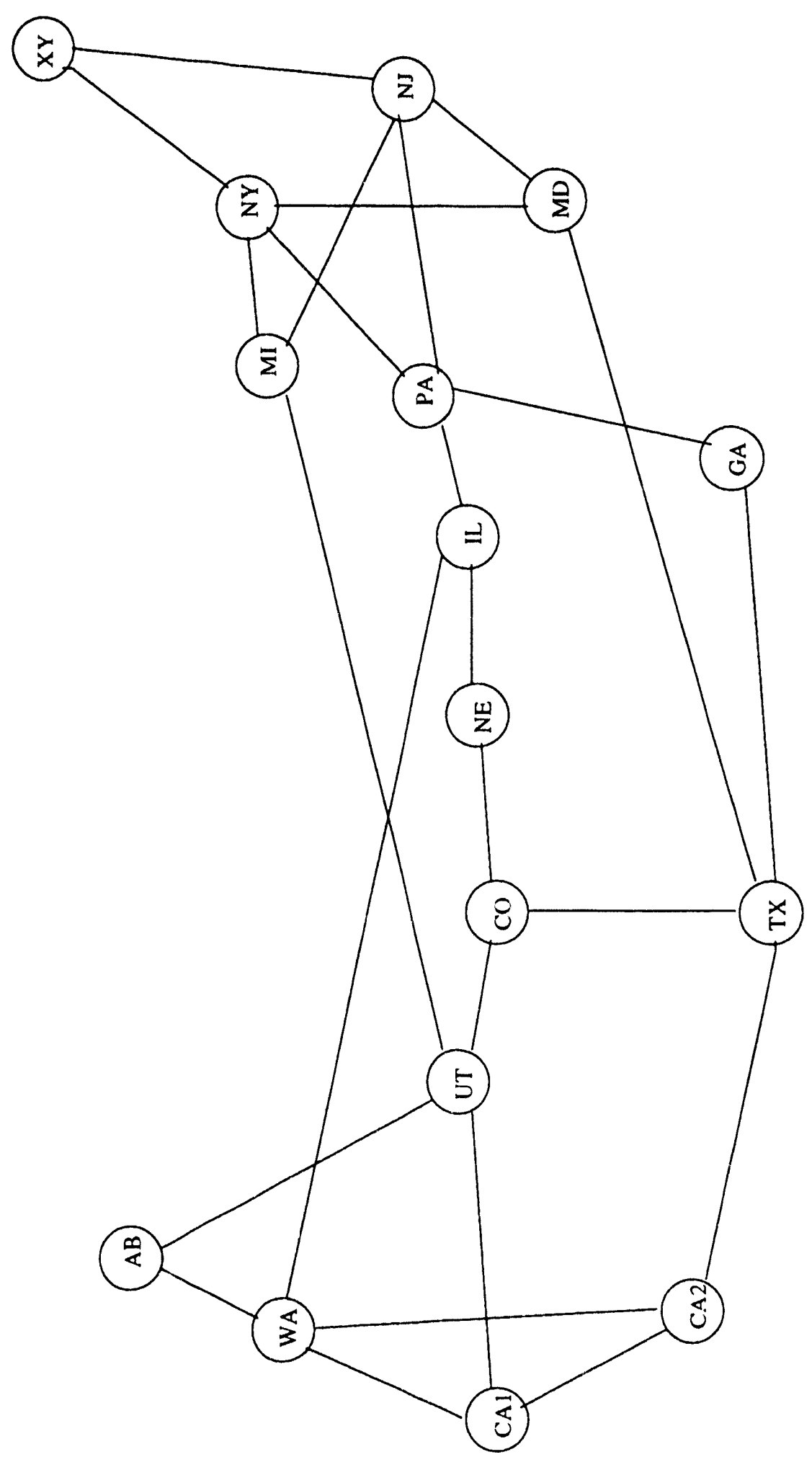

Figure 3: The NSF sample network 


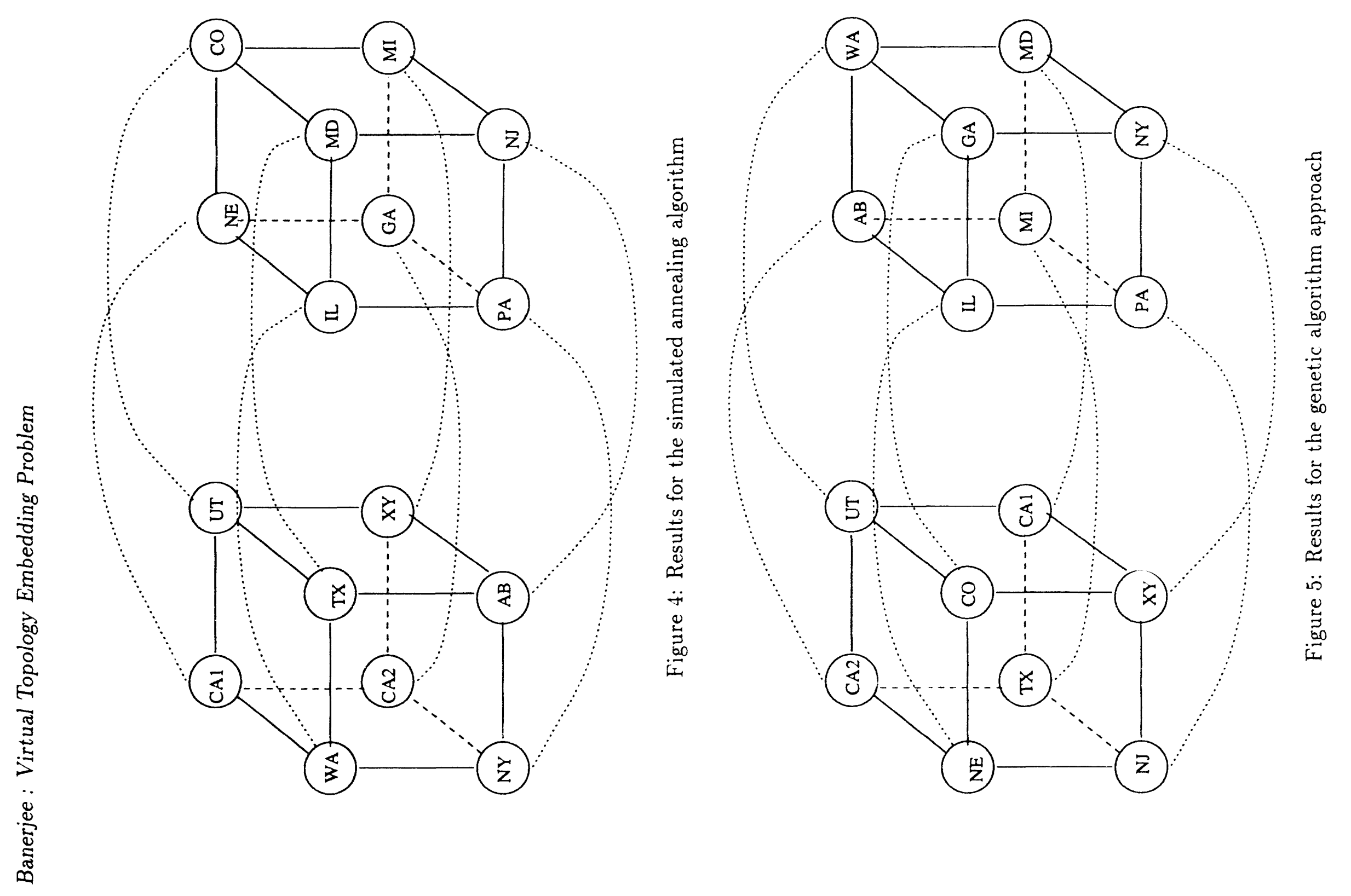




\title{
A Study of Genetic Algorithms to Find Approximate Solutions to Hard 3CNF Problems
}

\author{
Jeremy Frank \\ EAL 216 \\ frankâacs.ucdavis.edu \\ (916) 758.5925 \\ Division of Computer Science \\ University of California at Davis \\ Davis, CA. 95616 \\ December T. 1993
}

\begin{abstract}

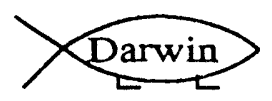

Genetic algorithms have been used to estimate optimal solutions to a variety of hard optimization problems ranging from the Quadıatic Assignment problem to the Travelling Salesman problem. A version of the standard algorithm called the Parallel Genetic Algorithm improves on the original. exploiting the explicit prarallelism of genetic algorithms by improving population diversity through separation of small iub. populations. and by employing hill-clinbing. We shall investigate both the simple and parallel genetic algorithms used to solve the 3 ('NF problem. We shall determine how effective genetic algorithms are in solving such problems.
\end{abstract}




\section{Introduction}

The Genetic Algorithm proposed by Holland has become the basis for a new approach to optimization problems. In a genetic algorithm, sets of solutions called populations are succeeded by new solutions which are closer to the desired optima. Criteria for creating successive generations from previous generatious are directly related to the function being optimized; solutions are selected on the basis of a fitness function related to the optimization function. The new population is then subject to genetic operators such as crossover and mutation [Go]. Genetic algorithms (hereafter called (iAs) have been tested on a wide variety of problems, including the combinatorially. difficult problems in the class NP. A typical NP-Hard problem is the Boolean Satisfaction Problem. where we desire an assignment of boolean variables which satisfies a boolean formula. One version of the Boolean Satisfaction problem is called $3 \mathrm{CNF}$. In this problem. terms of conjunctions of 3 distinct variables are disjoined in a formula. The optimization problem for CNF requires maximizing the number of true terms in the formula.

$$
\begin{gathered}
(\neg X \vee \neg Y \vee \neg Z) \wedge(X \vee Y \vee \neg V) \wedge \\
(X \vee \neg Y \vee \neg Z) \wedge(U \vee V \vee W)
\end{gathered}
$$

Figure 1: An example of a 3CNF problem.

Figure 1 show's a sample problem of this type.

Backtracking procedures are used to find exact solutions (or indicate that there is no solution) to $3 \mathrm{CNF}$ problems. Within this class of problem. if the ratio of terms to variables is close to 4.3 then these problems tend to be the most difficult to solve [MiSe]. Greedy local techniques, used to solved the optimization version of $3 \mathrm{CNF}$. show improvement over even the best backtracking methods for finding the solutions to solvable problems [Se]. [SeKa]. However, there are problems which can fool greedy algorithms into finding local optima unless the algorithms are specially modified [SeKa]. This brings to mind the so-called "deceptive" functions which are shown to fool GAs into finding suboptimal solutions. Indeed, some researchers feel that real-world problems are almost always deceptive [Mu].

To improve GAs performance on deceptive problems researchers have introduced parallel genetic algorithms. (referred to generally as PGAs). PGAs are designed to enhance genetic diversity by separating sub-populations. In some PGAs individuals are separated and only allowed to mate with certain neighboring individuals [Mu2]. while in other algorithms sub-populations are allowed to exchange individuals after a certain time period has elapsed [Ta]. Hill-climbing is introduced to improve some parallel algorithms. Performing crossover on local optima gives better results than crossover on other 
points, and hill-climbing improves individuals more quickly than the genetic operators [MuSc].

In this paper we will construct several GAs to solve the optimization version of the $3 \mathrm{CNF}$ problem. We shall analyze these GAs and investigate the settings of parameters such as crossover probability, mutation probability and population. We shall make use of a variety of performance measures to analyze the results of our algorithms. For the test suite, we propose to use randomly generated "hard" 3CNF problems of different sizes.

\section{The Genetic Algorithm and 3CNF}

To build a simple genetic algorithm (SGA) to solve the 3CNF problem we first investigate the encoding. In these problems, we desire a set of assignments to the variables of the boolean formula such that the maximum number of terms is satisfied. Thus a natural encoding is a string of 0 s and 1 indicating the truth assignment to each boolean variable indicated by the position of the bit in the string. The fitness function we use in this case is exactly the function to be optimized, the number of true terms for a particular assignment. Crossover and mutation naturally follow from such an encoding and objective function.

\subsection{The Hill-climbing Genetic Algorithm}

Hill-climbing algorithms operate by evaluating solutions in a neighborhood around the current proposed solution, and visiting the best neighboring solution. This process begins in an initially random state. and continues until a termination criteria has been met. For the $3 \mathrm{CNF}$ problem, solutions are simply assignments of values to the boolean variables. A natural neighborhood of solutions is those solutions differing in one bit assignment from the current solution. The termination criteria, selecting the next state to visit. and other details are dependent on the exact version of hill-climbing algorithm; among the options are gradient-descent (or ascent), tabu search [Gl]. simulated annealing and greedy local search [Se]. For this paper we shall use the gradient descent method described below. Here, the hill-climbing ceases when no state improves upon the current state.

In the HGA, hill-climbing is done before crossover and mutation; thus the HGA can be considered to be a genetic algorithm operating in the space of local optima for a given problem. It should be pointed out that there may still be an exponential number of optima in the search space. In cases where it is important to find as many local optima as possible, this method guarantees finding the optima and attempts to find better optima using the genetic operations. It may also be less expensive to use hill-climbing to do some of the work of the GA, thereby reducing the population size and/or the number of generations required to converge [Mu2]. 


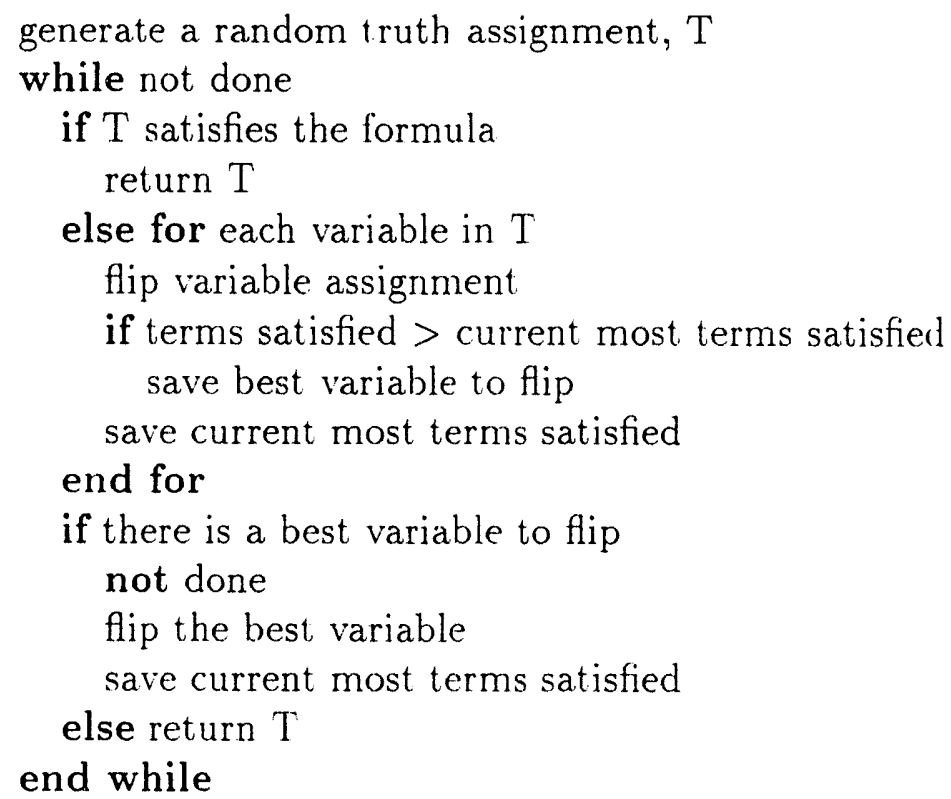

Figure 2: Gradient Descent Algorithm

\subsection{The Parallel Genetic Algorithm}

Genetic algorithms are just as suceptible to premature convergence to local optima as other search procedures. To address this problem, genetic algorithms induce diversification throughout the lifetime of the algorithm using parallel populations which exchange individuals for genetic crossover [Mu]. These algorithms are known as parallel genetic algorithms or PGAs. Wright argues that for PGAs to avoid local optima a PGA should be divicled into isolated sub-populations, one of which will eventually take over the others [Mu]. We take this view in designing a PGA to solve $3 \mathrm{CNF}$ problems. Accordingly, the PGA is divided into sub-populations with exactly two neighbors each arranged in a ring $[\mathrm{Mu}]$. Each sut.population sends a small number of randomly picked individuals to it's neighbors after a number of generations [Ta]. These individuals randomly replace members of the populations they are sent to. By only allowing a small number of "migrants" and by keeping the rate of migration small, we maintain diversity in the sub-populations, but allow opportunities for improvement by exchanging individuals. This algorithm can be modified by allowing hill-climbing.

\section{Analysis}

We ran a series of experiments to determine which algorithm is most appropriate for solving $3 \mathrm{CNF}$ problems. We asked the following questions:

- Which algorithm finds the best optima? 
- If a problem is solvable, which algorithms solve the problems the fastest?

- What are the effects of crossover and mutation?

- What are the effects of the migration rate and migration interval on parallel algorithms?

- What is the effect of the explicit parallelism on parallel algorithms?

- What is the effect of hill-climbing?

To answer these questions we created test suites of 100 randomly generated problems of size 25 and 50 variables. We ran our algorithms with carefully chosen parameters on these problems and analyzed the following results:

- The optima found by each algorithm for different sets of parameters

- The number of problems each algorithm solved, if any

- The average number of generations each algorithm took to solve the problems

- The average number of problems the algorithms showed improvement for; that is, the number of times the algorithm found a better solution after the first generation

\subsection{Generation and Population size}

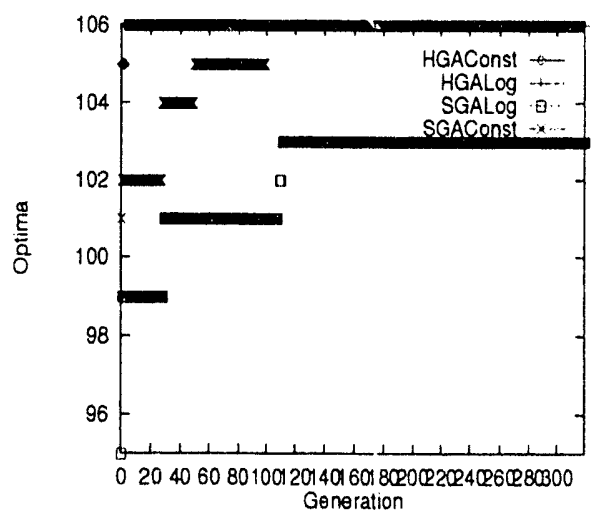

Figure 3: Execution of SGA and $\mathrm{PGA}$ on 25 variable $3 \mathrm{CNF}$

The population sizes and number of generations are motivated by the work of Goldberg [Go2]. We ran experiments using generations calculated for both the "logarithmic time convergence" and "constant time convergence" models. We selected a constant time of 100 generations, and used the time $100 \log n$ generations for the logarithmic model. We computed the optimal population size from Goldberg's function for both models, and selected bracketing values for the constant time convergence model. 
Figure 3 and 4 show sample executions of SGA and HGA under both models. In figure 3 we see the execution of these algorithms on unsolvable problems. Note that the initial optima found for the logarithmic model is smaller than that found for the constant model, and the final optima found is smaller than that found in the constant model. Also note that the values found for HGA are initially higher than those found in SGA.

Figure 4 shows the execution of these algorithms on solvable problems. The same behavior of the logarithmic and constant models is exhibited here. We see that SGA failed to solve the problems, but that one run of HGA did solve the problem. However, notice that one run of HCiA did not solve the problem, and in fact failed to show any improvement at all. This suggests that even HGA can be fooled, but also suggests that parameter tuning is more important for $\mathrm{HGA}$.

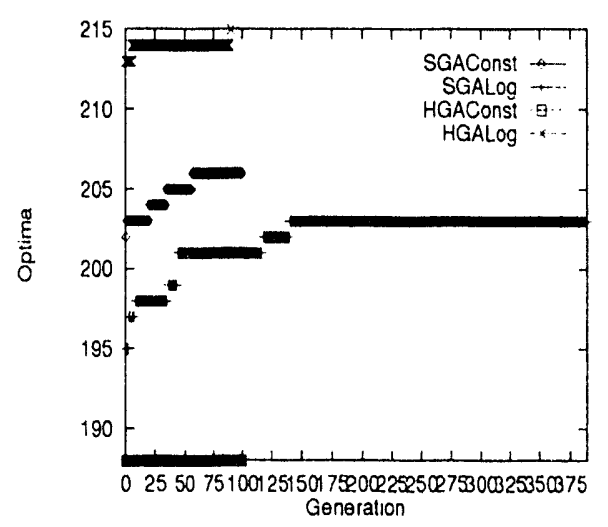

Figure 4: Execution of $\mathrm{SGA}$ and $\mathrm{PGA}$ on 50 variable $3 \mathrm{CNF}$

\subsection{Parallel Population Sizing}

The population ranges for the tests were difficult to motivate using Goldberg [Go2] since migration is not accounted for. We decided to igriore the effects of migration and use Goldberg's function to calculate the population sizes for selected values of $\beta$. Using the formula $n^{\beta}=d$ we solved for the number of parallel subpopulations. The results of these calculations were modified so that the sub-population sizes obeyed other constraints. For instance, the number of sub-populations must evenly divide the population size and result in sub-populations with an even number of chromosomes.

\subsection{Migration Rate and Migration Interval}

We desire to keep the migration rate and interval low enough to keep diversity high. The migration rates were set at . 5\%, 10\% and $25 \%$ of the sub-population sizes. We limited the migration interval such that information cannot propagate further than a fixed distance around the ring. We allow information to 


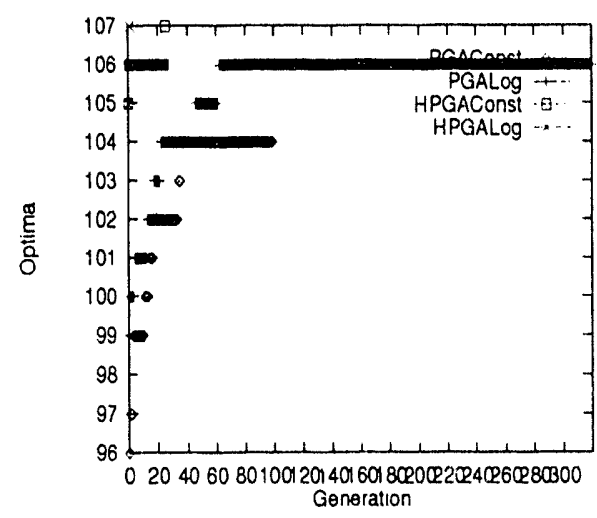

Figure 5: Execution of PGA and HPGA allowing half-ring travel

travel $1 / 2$ the ring, all the way around the ring, and around the ring twice. In cases where this was not possible due to other constraints we chose values as close to these as possible. We also fixed the mutation rate at .01 and the crossover rate at .25 for the parallel algorithms. Here, we were much more interested in the effectiveness of migration than in these parameters.

Figures 5 and 6 show the execution of PGA and HPGA with migration rates allowing information to travel around $\frac{1}{2}$ way around the ring and twice around the ring. Again we see that the values for HPGA exceed those found in PGA, that under the constant model we find smaller optima, and that HPGA solves the problem where PGA does not. We hope to see improvement in the optima found by these algorithms after a migration is allowed. In figure 5 the migration intervals are every 16 generations for the logarithmic model and every 50 generations for the constant model. There is some evidence that the logarithmic model shows improvement roughly around the times of migration. In figure 6 the migration intervals are every 10 generations for the constant model and every 4 generations for the logarithmic model. There is strong evidence of improvement after migration for both models.

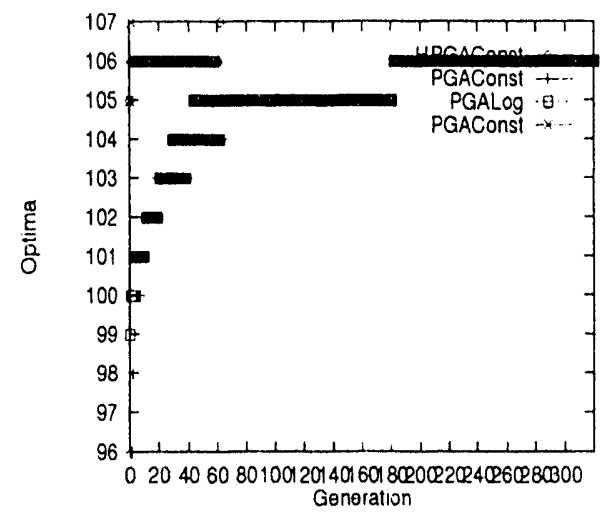

Figure 6: Execution of PGA and HPGA allowing twice-ring travel 


\subsection{Crossover and Mutation}

For all algorithms we selected crossover rates of .25. .35 and .45. We desired a reasonable amount of recombination while allowing for some repetition in members of the population. Since 3C'NF problems contain many local optima, we felt that large crossover rates would move the initial points used for hillclimbing enough to obtain a good sampling of new optima. For all runs we chose mutation rates of $.01, .03$ and .05 .

\subsection{Results}

For SGA, our results were surprisingly similar: most parameter settings failed to solve any of the problems of either 25 or 50 variables. The mean optima found was better in the constant model than in the logarithmic model; this would seem to be accounted for by the larger population size. The rate of improvement was better in the logarithmic model than in the constant model for the problems of 25 variables, but for 50 variables the run with population 2000 performed better. The only consistent change in performance was in the standard deviation of optima found. The standard deviation dropped with the rising mutation rate consistently. This indicates a higher mutation rate should improve performance in future evaluations.

\begin{tabular}{|c|c|c|c|c|c|c|}
\hline \multicolumn{7}{|c|}{ SGA } \\
\hline Vars & Model & Gens & Popsize & Mean Opt & Sdev Opt & 9 Iniproved \\
\hline 25 & $\log$ & 320 & 4 & $101-102$ & $10-16$ & $93 \%$ \\
\hline 25 & const & 100 & 30 & 103 & $10 \cdot 12$ & $88 \%$ \\
\hline 25 & const, & 100 & 40 & $103-104$ & $9-11$ & $88 \%$ \\
\hline 25 & const & 100 & 50 & $103-104$ & $9-12$ & $89 \%$ \\
\hline 50 & $\log$ & 390 & 4 & $200-202$ & $14-20$ & $95 \%$ \\
\hline 50 & const & 100 & 1500 & $206-207$ & $10-13$ & $\overline{94 \%}$ \\
\hline 50 & const & 100 & 2000 & 207 & $10-13$ & $98 \%$ \\
\hline
\end{tabular}

For HSGA the results were rather different. HSGA solved most (if not all) of the solvable problems, and thus the mean optimum is close to identical for all parameter settings. Interestingly the standard deviations are also close to identical for the $\log$ and constant models, indicating that the hill-climbing component does most of the work and that the genetic operators did little to enhance the search. Again, the standard deviation dropped with an increase in the mutation rate, and also appeared to drop with crossover. The number of problems solved improved in the constant model. and was slightly higher on average in the constant model for 25 variables. The mean time to solution for those solvable problems was low, but most problems were solved in 1 or 2 generations with only a few examples taking longer. Finally, HSGA failed to improve solutions beyond the 1st generation consistently, and even improved less often the larger the initial population. Even for those problems in which 
HSGA found a better solution after generation 0 , it typically did so very early and failed to improve the situation more than twice throughout the entire run.

\begin{tabular}{||l|l|l|l|l|l|l||}
\hline \multicolumn{7}{|c||}{ HGA } \\
\hline 25 & Model & Gens & Popsizc & $\%$ Improved & Solved & Mean time \\
\hline 25 & const & 100 & 6 & $48 \%-64 \%$ & $48-64$ & 5 \\
\hline 25 & const & 100 & 8 & $25 \%-44 \%$ & $53-66$ & 4 \\
\hline 25 & const & 100 & 10 & $33 \%-41 \%$ & $56-67$ & 5 \\
\hline 50 & $\log$ & 390 & 4 & $45 \%-56 \%$ & $48-56$ & 5 \\
\hline 50 & const & 100 & 150 & $39 \%-47 \%$ & $47-53$ & 5 \\
\hline 50 & const & 100 & 200 & $32 \%-45 \%$ & $46-54$ & 4 \\
\hline
\end{tabular}

In PGA we see that employing migration does make a difference. PGA solved problems where SGA did not; this is not surprising given the significantly larger populations. Furthermore, PGA improved upon the optima in the initial population $100 \%$ of the time, which may also be due to the larger initial population. What is surprising is that the mean time to solution showed a decrease with the rate of migration. This implies that larger migration rates improve the performance of PGA on $3 \mathrm{CNF}$ problems. There was no discernable effect of changing the migration rate on the overall performance of PGA. This may be due to choosing small migration rates. The mean and standard deviation for the optima found did not vary with the parameters.

\begin{tabular}{|c|c|c|c|c|c|c|}
\hline \multicolumn{6}{|c|}{$\overline{\mathrm{PGA}}$} & \\
\hline Model & Gens & Popsize & Subpops & $\%$ Improved & Solved & Mean time \\
\hline $\log$ & 320 & 4000 & $25(27.5)$ & $100 \%$ & $35-40$ & $106-143$ \\
\hline const & 100 & 2000 & $4(4.5)$ & $100 \%$ & 11 & $59-65$ \\
\hline const & 100 & 10000 & 40 & $100 \%$ & $16-22$ & $58-65$ \\
\hline
\end{tabular}

For HPGA the results are similar to those for PGA. Here, the number of problems solved rises slightly with the migration rate, and the mean time decreases slightly with migration rate or does not vary. The rate of improvement also increases slightly with the migration rate. We also see the percentage improved go down with larger population size, as in HGA above. Again, the mean and standard deviation for the optima found did not vary with the parameters. 


\begin{tabular}{||l|l|l|l|l|l|l||}
\hline \multicolumn{7}{|c||}{ HPGA } \\
\hline Model & Gens & Popsize & Subpops & $\%$ Improved & Solved & Mean time \\
\hline $\log$ & 320 & 800 & 16 & $56 \%$ & 67 & 5 \\
\hline const & 100 & 400 & 4 & $56 \%-58 \%$ & $65-67$ & $4-5$ \\
\hline const & 100 & 2000 & 20 & $14 \%$ & 67 & $2-3$ \\
\hline
\end{tabular}

\section{Summary and Conclusions}

We have adapted the genetic algorithm to solve the optimization version of the $3 \mathrm{CNF}$ problem using both parallel and hill-climbing techniques. It is clear that fir SGA a larger mutation rate is desirable, and possibly higher crossover rates as well. It is also clear that larger populations give better results, thus Goldberg's constant model for convergence ought to be used in the future. Finally, for serial algorithms, hill-climbing algorithms gave the best results for both optima found and solving problems. In the parallel algorithms, we saw that migration rate certainly plays a factor in solving $3 \mathrm{CNF}$ problems. but that migration rate did not necessarily play a role. We explain this by concluding that more work must be done to identify the effectiveness of migration rate in solving these problems. We also conclude that hill-climbing algorithms perform better than non- hill-climbing algorithms.

A great deal of future work remains to be done in this area. We should test other hill-climbing methods such as tabu search and GSAT in HGA. In PGA we currently select migrants and replace elements of the new populations at random. A wide variety of selection schemes should be tested and compared. For the HGA and the HPGA we should select higher crossover and mutation rates than for SGA and PGA. Since the algorithms employ hill-climbing, we may desire a more vigorous exploration of points in an attempt to find different local optima in our search.

\section{References}

[ChKa] P. Cheeseman, Bob Kanefsky, William Taylor. "Where the Really Hard Problems Are." Proceedings of the $12^{\text {th }}$ IJCAI. 1991.

[MiSe] D. Mitchell, B. Selman, H. Levesque. "Hard and Easy distribution of SAT Problems." Proceedings, AAAI, 1992. pp. 459-465

[SeKa] B. Selman, H. Kautz. "An Empirical Study of Greedy Local Search for Satisfiability Testing." Proceedings, AAAI Spring Symposium on Artificial Intelligence and NP-Hard Problems, 1993. pp. 149-155.

[Gl] f. Glover. "Tabu Search for Nonlinear and Parametric Optimizations". Discrete Applied Mathematics, 1991 
[Go] D. Goldberg. Genetic Algorithms in Search, Optimization and Machint Learning. Menlo Park, Wiley and Sons, 1989.

[Go2] D. Goldberg. "Sizing Populations for Serial and Parallel Genetic Algorithms." Proceedings, $3 d$ International Conference on Genetic Algorithms, 1989.

[Se] B. Selman. "A New Method for Solving Hard Satisfiability Problems." Proceedings, AAAI, 1992, pp. 440-4.t6.

[MuSc] H. Mülenbien. M. Schomisch. J. Born. "The Parallel Genetic Algorithm as a Function Optimizer." Proceedings, 4th International Conference on Genetic Algorithms, 1991, pp. 271-278.

[Mu] H. Mülenbien. "Evolution in Time and Space: The Parallel Genetic Algorithm." In Foundations of Genetic Algorithms. G. Rawlins. ed., Morgan Kaufman. 1991.

[Mu2] H. Mülenbien. "Asynchronous Parallel Search by the Parallel Genetic Algorithm." Proceedings, 3d IEEE Symposium on Parallel and Distributed Systems, 1992. pp. 526-533.

[LiBa] G. Liepens, S. Baluja. "apGA: An Adaptive Parallel Genetic Algorithm." In Computer Science and Operations Research, Neu Developments in Their Interfaces. Balci, Sharda. Zeinos, ed.. Pergamon Press. 1992.

[Ta] R. Tanese. "Distributed Cienetic Algorithms." Proceedings, 3d International Conference on Genetic Algorithms. 1989. 


\title{
Appendix: Relevant Course Materials
}

\author{
EAL 216G Genetic Algorithms: Handout 1, Page 1 \\ Instructor: V. Rao Vemuri \\ Address: P. O. Box 808, L-794 \\ University of California at Davis \\ Livermore, CA 94550 \\ E-mail:Vemuri@icdc.IInl.gov \\ Telephone: (510) 424-4051 \\ FAX: (510) $422-8681$
}

Class Times: Tu, Th: 12 to $1.30 \mathrm{pm}$

Class Rooms:

Livermore:

Room 13 Hertz Hall on Thurs. (XMIT)

Room 10 Hertz Hall on Tues. (RECV.)

Davis:

Room 1062, Bainer Hall on Tues. (XMIT)

Room 1122, Bainer Hall on Thurs. (RECV.)

Office Hours:

Livermore:

Room 18, Hertz Hall 1.30 to 2.30 Th.

Davis:

Room TBD, Bainer Hall 1.30 to 2.30 Tu.

Text Book:

${ }^{\circ}$ Goldberg, D. Genetic Algorithms in Search,

1989.

Optimization and Machine Learning, Addison Wesley,

${ }^{\circ}$ Copies of the book are available with Estelle Miller in the Livermore office.

Reading List: Will be supplied separately.

Grading:

$20 \%$ for home work 
$40 \%$ for term paper

$40 \%$ for final examination

Key Dates: (first cut)(will be revised soon)

First Lecture:

30 Sept. 1993; Thursday

Submit project proposals: 21 Oct. 1993; Thursday

Project proposal approved: 28 Oct. 1993; Thursday

Projects Due:

7 December; Thursday

Take Home Final:

7 December; 1993

(consisting of peer review

of three projects from other

students distributed)

Take Home Final Due: 14 December, 1993

Lecture Schedule (Subject to continuous updating)

Date: Reading Assignment

(To be read PRIOR to the class)

30 Sep.

5 Oct. Problem sets 1,2, 3 given

7 Oct. Chapter 1, Appendix A

12 Oct. Problem sets 1,2 due Chapter 3

14 Oct. $\quad$ Chapter 2, pages 27-54

19 Oct. Problem set 3 due

project proposals due

21 Oct.

26 Oct. Walter Cedeno lecture

Project proposal approved

28 Oct. Chapter 4, pp. 99-120, 126-129, 136-142

7 Dec. $\quad$ Projects Due; 2.00 pm

7 Dec. Take Home Finals distributed by $2.00 \mathrm{pm}$ 
Friday at both locations on Friday

$14 \mathrm{Dec} \quad$ Take Home finals are due by $2.00 \mathrm{pm}$

Computer Programs:

1. I gave you an ftp address from where you can get some $C$ programs. Feel free to use one of them.

2. Feel free to key in the SGA program (Pascal) from the text book!!!

3. I am placing a version of the SGA Program (Pascal) in one of the LLNL computers. This version is almost the same as the program in the text book with a couple of modifications made by Prof. Koza of Stanford. You can ftp that program if you wish.

Directory name is: pub/cedeno

Program name is: $\quad$ sga.pas.tar. $Z$ (the $Z$ is upper case)

4. I am placing a version of a C Program in one of the LLNL computers. This is written by Walter Cedeno. This program allows you to play around with the De Jong test functions shown on pages 108-110 of your text book.

Directory name is: pub/cedeno

Program name are: galib.tar. $Z$ (the $Z$ is upper case)

testga.tar.Z

misclib.tar.Z

DONOT WASTE YOUR TIME SWITCHING BACK AND FORTH. SETTLE ON ONE PROGRAM, UNDESTAND IT WELL, AND ADAPT IT TO MEET YOUR NEEDS.

References:

1. Proceedings of all the International Conferences on Genetic Algorithms, Morgan Kaufmann, San Mateo, CA

2. Michalewicz, Zbignew, Genetic Algorithms + Data Structures = Evolution Programs, New York: Springer Verlag, 1992. 


\section{EAL 216G (Genetic Algorithms and Optimization) Project FORMAT OF PAPERS FOR TERM PROJECTS AND TAKE- HOME FINAL CONSISTING OF PEER REVIEW OF PAPERS}

DEADLINE FOR YOUR PAPER: 7th Dec. 93 THIS DEADLINE IS NOT EXTENDIBLE. FORMAT AND STYLE: Please bring six stapled copies of your paper to class (no binders of any kind). The paper is not to exceed 10 pages. The format is single spaced, 12-point type, $8.5 \times 11$ inch paper, $1 "$ margins at top, bottom, left and right. The ten page limit includes everything (except the special attachment of your program listing described below). That is, this ten page limit includes all the usual limits of a technical paper for a journal or a conference, namely the title, author's name and address, the abstract (75 to 150 words), full text of the paper, headings, subheadings, figures (if any), tables (if any), acknowledgments (if any), the bibliography, and the appendix (if any).

Your paper can use any consistent style commonly used in technical papers and reports. Do not waste too much time on cosmetics. Just adopt a style and use it consistently. In citing references, please use the author-date style (Smith, 1987) rather than the consecutive numbering style. Your paper should begin with the title, author's name, full physical address, e-mail address, phone number and the abstract.

SPECIAL COPY WITH PROGRAM LISTING: A listing of your computer program (if any) should be attached to one of the copies of the paper. This listing is for the instructor. This one copy should be conspicuously labeled, in big letters, "CONTAINS PROGRAM LISTING" at the top of the first page. Your paper should stand alone and should not refer to this program listing in any way. If you wish to discuss a specific part of the program, then that part of the program must be entirely contained within your paper (i.e., as a table, a figure, running text, or appendix) and it will count as a part of the ten page limit.

SPECIAL SINGLE-SIDED COPY: At least one copy of your paper must be single sided. If we can arrange to bind the papers in a book form, this is the copy that will be used for that purpose. This special copy is distinct from the copy containing the program listing.

PROCEDURE: The copy containing the program listing goes to me for grading. The single sided copy will be set aside for possible future binding. The remaining copies will be sorted, during the class, into random batches, each batch containing 4 papers (not, your own of course). The batches will be distributed to each student submitting a paper. Also, each student gets four blanks of individual peer review forms and one blank of a overall review form. The individual review forms and the overall review form constitute the take-home examination.

THE PEER-REVIEW PROCESS: Start by reading the four papers in your batch. The individual peer review forms ask you to make specific comments on the strengths and weaknesses of each paper (viewed alone) in much the same manner researchers are asked to evaluate papers submitted for publication in technical journals and for presentation at conferences. In addition, the overall review paper asks you to give overall comparative ranking of the four papers and give specific reasons for your ranking (i.e., $A$ is better than $B$ because..., $B$ is better than $C$ because...) Your reviews will be graded in terms of the quality of the specific reasons you give on the individual ranking and on the overall ranking. Comments like "good paper" are of no interest. We are interested in specific comments supported by specific reasons - whether they are positive, negative or mixed. Keep the four copies you received.

RETURN OF PAPERS: One copy of the paper will be returned to you along with the comments received from your peers. The instructor will keep one copy for his files.

POSSIBLE BINDING: We will explore the possibility of binding these into a book form for distribution. If so, unless you explicitly specify otherwise (by typing "NOT FOR COPYING" on the top line of the first page of your paper), we will include your paper. The book will not be 
copy righted and the book will specifically note that each student retains all rights to his or her paper. A few extra copies may be made available for distribution or for sale by the bookstore, to partially recover the cost of reproduction.

\section{EAL 216G (Genetic Algorithms and Optimization) Take-Home Final}

This take-home final is to be submitted in a sealed envelope to the secretary in the Department of Applied Science, in Walker Hall, between $9 \mathrm{am}$ and $1 \mathrm{pm}$ on Dec.14th. This examination involves your reviewing three papers written by other students in the course of their descriptions of their own projects. This peer review process resembles the process by which researchers in the academic, commercial, government, and R\&D worlds are asked to evaluate papers submitted for publications in technical journals and books and for presentation at technical conferences. Different papers will have different strengths and weaknesses in different areas. Yet, they must all be ranked. There is no one correct way to review papers. Different reviewers will reach different conclusions for different reasons as to the ranking of the papers. The required process of ranking the three papers in linear order (no ties) requires the application of judgment involving many conflicting factors (supported of course by your specific reasons). Start by reading the papers in your specific batch. The three individual paper review forms attached hereto ask you to make comments on the strengths and weaknesses of each paper (viewed alone). The one overall review form asks you to make an overall comparative ranking of the three papers and give specific reasons for your ranking. (e.g. Paper \#1 is better than Paper \#2 because...) Keep in mind that your reviews will be graded in terms of the specific reasons you give on each of the individual papers and the reasons you give in the overall ranking. Unexplained comments, such as "good paper" are of no interest. We are inierested in specific comments with specific reasons (good, bad, or mixed). Thus, it does not matter whether the randomly selected group of three papers you got are unusually good or unusually bad. The quality of your reasons is what that matters, not the "intrinsic merit" of the papers you are reviewing. You are expected to do this work alone, without seeking help from any one, and the UC Davis Honor Code applies to this examination. 
EAL 216G (Genetic Algorithms and Optimization) Take-Home Final

Your Name:

\section{Overall Ranking of the 3 Papers}

My ranking of the 3 papers, (identified by author):

Best Paper:

2nd Best Paper:

Worst Paper:

Reasons for the above Ranking (an small essay) 
EAL 216G (Genetic Algorithms and Optimization)

Take-Home Final

Your Name:

\section{Evaluation of Individual Paper}

This page refers to the paper written by

1. Writing Quality: How good is the organization, sentence structure, spelling, mechanical construction, and above all, clarity of the paper?

2. Identification of the Problem. Does this paper clearly identify an issue to be resolved?

3. Coherence/ Achievement of stated Objective or asserted conclusion: Does this paper actually provide an answer (or partial answer) to the issue raised? Or did it, in fact, do something entirely different from what the author claims? 
4. Replicability: If an experiment is involved, (as will be the case with almost all of the termpapers), is there sufficient information in this paper to permit replication of the experiment? Why? If not, what is missing? This is the SECOND MOST IMPORTANT question in this group.

5. Suggestions to the Author: What suggestions would you make to improve on this paper? (NOTE: This question is almost always a part of evaluations, whether your paper is headed for acceptance or rejection.) This is THE MOST IMPORTANT question in this group. 


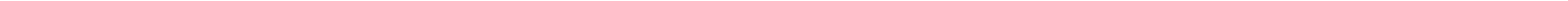




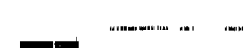

